\title{
Clinical applications of cardiac CT angiography
}

\author{
Ethan J. Halpern
}

Received: 6 July 2010 /Revised: 22 July 2010 / Accepted: 29 July 2010 / Published online: 13 August 2010

(C) European Society of Radiology 2010

\begin{abstract}
ECG-gated multislice CT provides a costeffective, non-invasive technology for evaluation of the coronary arteries, as well as for additional clinical applications, which require morphological assessment of the heart and adjacent structures with simultaneous evaluation of the coronary circulation.

The excellent negative predictive value of a normal coronary CTA (cCTA) examination excludes the presence of significant coronary disease in the symptomatic patient. Triple rule-out studies provide evaluation of the aorta and pulmonary arteries without loss of image quality in the coronary circulation. The ability to visualize surrounding vascular structures along with the coronary arteries is essential in the evaluation of coronary anomalies.

Cardiac CTA is useful in non-coronary applications, including evaluation of the thoracic aorta, cardiac valves and other aspects of cardiac morphology that may require surgical or percutaneous repair. Although radiation exposure is a limitation of cCTA relative to echocardiography and MRI, recent technological advances allow coronary imaging with effective doses as low as $1 \mathrm{mSv}$.

Recent advances in evaluation of coronary plaque morphology as well as myocardial perfusion will allow a more complete noninvasive cardiac assessment in the future and may provide a highly effective method of cardiac risk stratification to facilitate preventive cardiac care.
\end{abstract}

E. J. Halpern $(\bowtie)$

Department of Radiology,

Thomas Jefferson University,

132 South 10th Street,

Philadelphia, PA 19107-5244, USA

e-mail: Ethan.Halpern@jefferson.edu
Keywords CT angiography · Cardiac imaging

\section{Introduction}

The advent of multislice CT technology with ECG-gating along with innovations in x-ray tube and CT detector technology have resulted in revolutionary progress in cardiac imaging. ECG-gated CT angiography with 64+ slice systems has demonstrated efficacy for the diagnosis of coronary disease, cardiac morphology, and valvular assessment. Recent studies suggest additional applications of CT for assessment of myocardial perfusion and cardiac event risk. Among the clinical applications of cardiac CT, coronary $\mathrm{CT}$ angiography (cCTA) remains the most widespread and most controversial. Numerous studies suggest that cCTA is a cost-effective alternative to the nuclear stress test and diagnostic cardiac catheterization. Although CT angiography has replaced conventional arteriography for most visceral applications, issues related to radiation exposure and functional versus anatomical information have been raised as objections to widespread adoption of cCTA. Recent advances in CT imaging methodology and CT image reconstruction algorithms address many of these concerns.

This review explores a variety of clinical applications of cardiac CT. The primary focus is on cCTA with a review diagnostic accuracy, appropriate clinical indications and limitations for evaluation of the coronary arteries. Although the principle indication for cardiac CT is most often for evaluation the coronary arteries, cCTA is often requested for the dual purposes of non-coronary and coronary evaluation. Several of the more common non-coronary applications are reviewed, although a complete review of these applications is beyond the scope of this pictoral essay. 
Fig. 1 Volumetric rendering of a left dominant coronary circulation from cCTA. a Left anterior oblique view demonstrates the left anterior descending artery coursing down the anterior interventricular groove (black arrowhead) and the circumflex artery coursing in the left atrioventricular groove (white arrowhead). b View of the base of the heart from the left side demonstrates the continuation of the circumflex artery in the posterior left atrioventricular groove (arrowhead), extending to the crux of the heart where it continues as the posterior descending artery (white arrow)
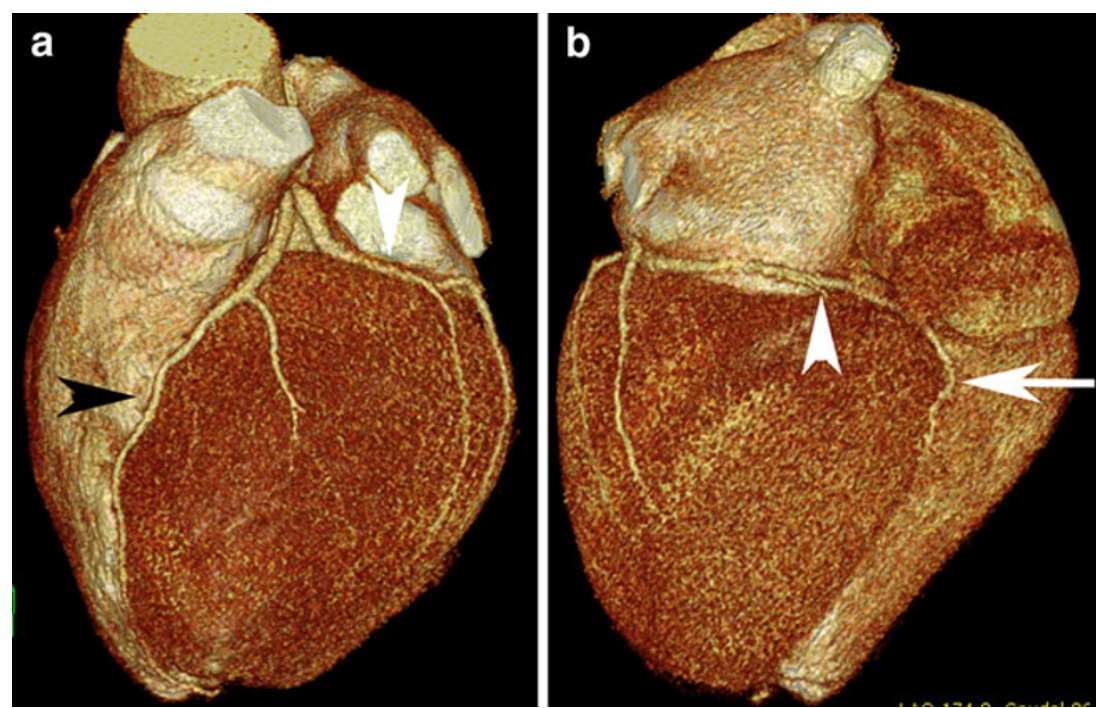

\section{Coronary Artery Disease (CAD)}

Despite clinical advances in diagnosis and treatment, heart disease remains the leading cause of death for both men and women, and is responsible for $26 \%$ of all deaths in the United States [1]. Atherosclerotic disease of the coronary arteries remains the most important etiology of heart disease. Coronary computed tomographic angiography (cCTA) provides a non-invasive alternative to cardiac catheterization for direct visualization of coronary anatomy (Fig. 1). Recent meta-analyses of cCTA studies have demonstrated high sensitivity (96-99\%) and specificity (88-91\%) for the anatomic presence of CAD [2-5]. Although functional information about wall motion can be obtained during cCTA, the primary utility of cCTA is for imaging of the coronary arteries to define the presence of CAD (Figs. 2, 3, 4). The high sensitivity of cCTA for CAD results in an extremely high negative predictive value, especially in patients with low to intermediate risk of coronary disease. A negative cCTA study in this population effectively excludes the diagnosis of CAD. Although the specificity of cCTA is limited by arterial calcification, post-processing techniques using vessel tracking and curved multiplanar reconstructions are often useful for evaluation of calcified vessels (Fig. 5). Nonetheless, blooming artifact associated with densely calcified plaque may preclude visualization of the underlying vascular lumen in the absence of significant stenosis (Fig. 6).

Whereas cCTA provides an anatomic depiction of coronary stenosis, stress testing provides additional information to predict the functional relevance of coronary stenosis $[6,7]$. When cCTA demonstrates no more than minimal disease of the coronary arteries or when cCTA demonstrates the presence of high-grade coronary stenosis, the clinical implication is clear. When cCTA demonstrates coronary stenosis of uncertain hemodynamic significance, a
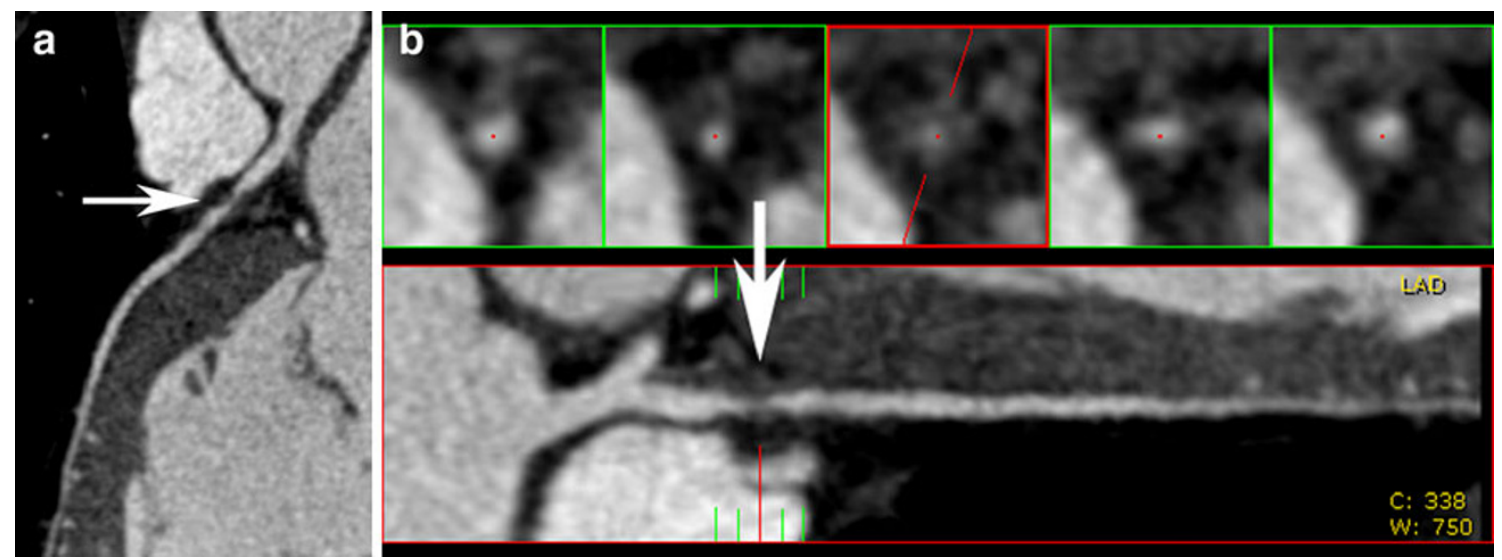

Fig. 2 Severe stenosis of the proximal left anterior descending artery (LAD) in a patient presenting with chest pain. a Curved MIP demonstrates greater than $70 \%$ narrowing in the proximal LAD (arrow). b Straightened lumen MIP again demonstrates severe stenosis of the proximal LAD (arrow). Cross-sectional images of the LAD are presented above the straightened vessel view. The lumen disappears in the middle image at the site of maximum narrowing 

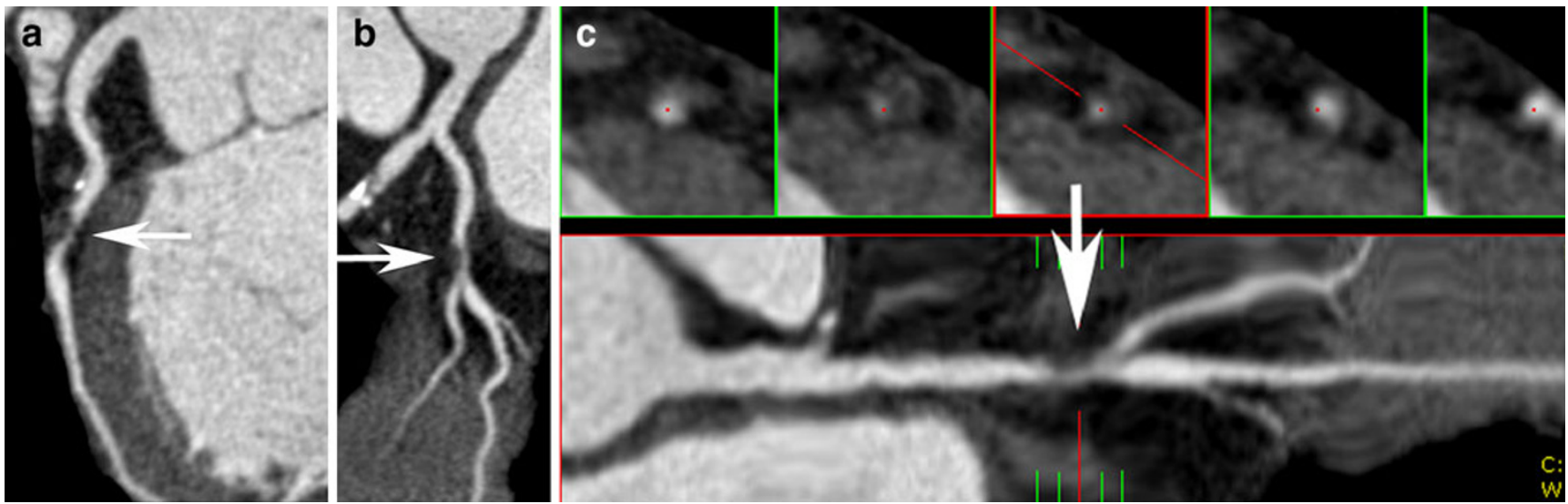

Fig. 3 Severe stenosis of the proximal circumflex artery (LCX) in a patient complaining of chest pain. a Curved MIP demonstrates greater than $70 \%$ narrowing in the proximal LCX (arrow). b Curved MIP in an orthogonal projection again demonstrates greater than $70 \%$

stress test may be useful to evaluate the functional/clinical significance of CAD found by cCTA. This difficulty in defining a "clinically relevant" stenosis is also a limitation of the "gold standard" conventional arteriography, which demonstrates the degree of luminal narrowing and can provide measurements of pressure gradients but does truly evaluate the impact of coronary stenosis on myocardial perfusion. In the setting of moderate CAD, cCTA and stress testing may provide complementary information for the evaluation of CAD [8].

A recently published decision analytic model for evaluation of the symptomatic patient with chest pain demonstrates how cCTA may be used to improve diagnostic accuracy, reduce the effective radiation dose and limit overall imaging costs for the evaluation of CAD [9]. A workup strategy that employs stress ECG or stress echocardiography prior to cCTA minimizes effective radiation exposure compared to all other strategies that use cCTA and/or narrowing in the proximal LCX (arrow). c Straightened lumen MIP confirms the severe stenosis just proximal to the first obtuse marginal branch (arrow)

nuclear perfusion scanning. When the pre-test probability of CAD is below $10 \%$, a strategy that employs stress echocardiography followed by cCTA before proceeding to cardiac catheterization will result in an effective radiation dose that is lower than a strategy that uses stress echocardiography followed by conventional cardiac catheterization. Imaging costs are minimized by a strategy that employs stress ECG or stress echocardiography with follow-up by cCTA for positive stress tests, as the cCTA can identify false-positive stress tests and eliminate many negative diagnostic cardiac catheterization procedures.

The most appropriate testing strategy for the workup of symptomatic CAD depends upon the pre-test probability of disease, which should be estimated on the basis of demographics and clinical presentation [10]. The symptomatic patient with a high pre-test probability of CAD $(>50 \%)$ may benefit from direct triage to cardiac catheterization. For the patient with a low pre-test probability of
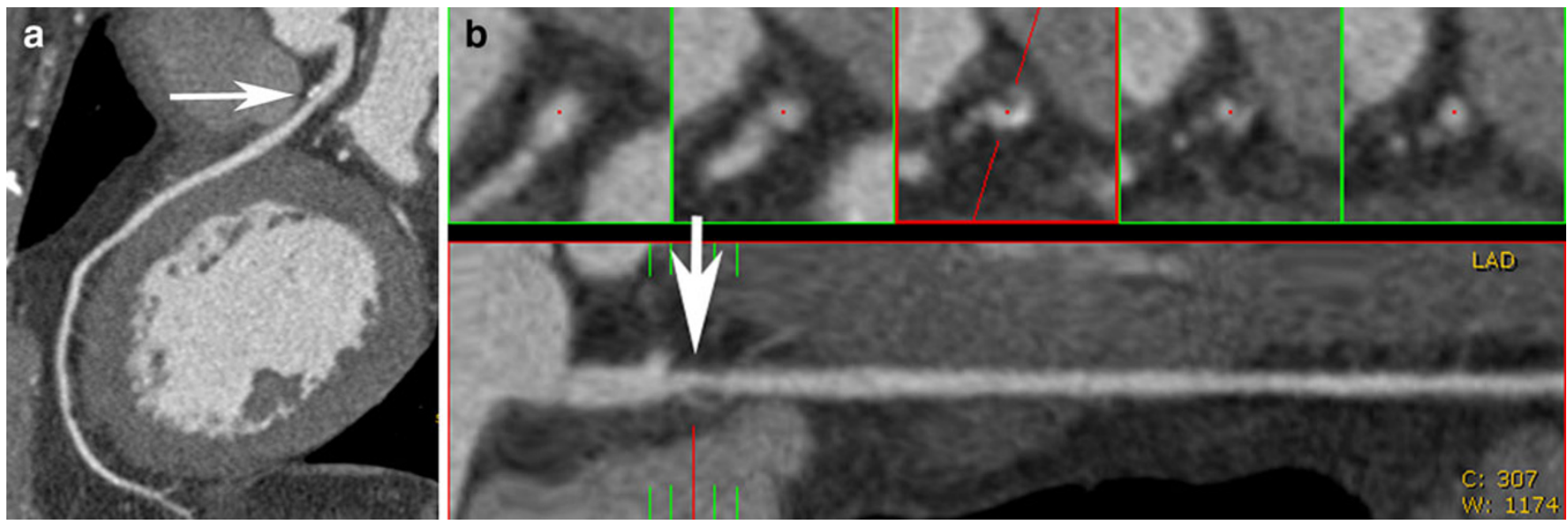

Fig. 4 Moderate stenosis of the proximal left anterior descending artery (LAD). a Curved MIP demonstrates calcified plaque in the proximal LAD (arrow). b Straightened lumen MIP demonstrates the presence of non-calcified plaque at this level with diameter reduction in the range of $50-70 \%$ 
Fig. 5 Minimal calcified plaque in the proximal left anterior descending artery (arrowheads) with no significant stenosis. Although the calcified plaque overlies the vessel in the globe MIP view (bottom right), the curved MIP and straightened lumen views are rotated to project the calcium off the vessel lumen and demonstrate that there is no significant stenosis

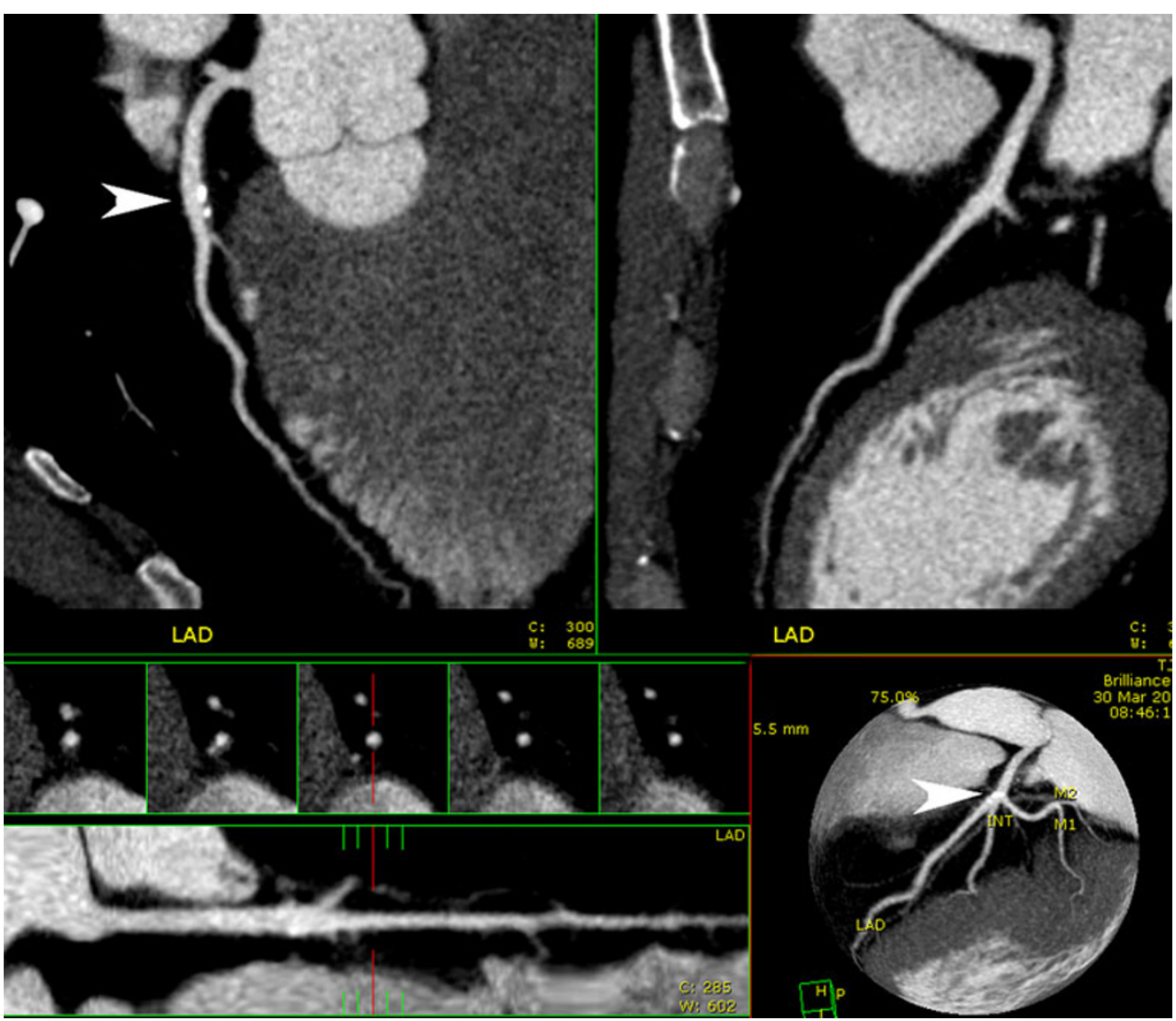

disease $(\leq 20 \%)$, the combinations of stress $\mathrm{ECG}+\mathrm{cCTA}$ or stress echocardiography + cCTA provides optimal options as judged by cost and radiation dose, while stress echocardiography + cCTA will result in the lowest rate of negative cardiac catheterization. As the pre-test probability of CAD is
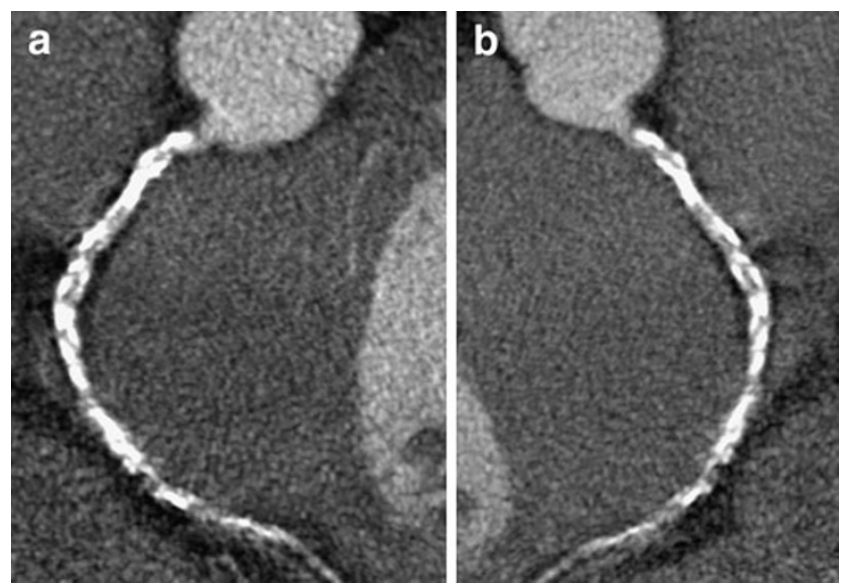

Fig. 6 Heavily calcified plaque in the right coronary artery. a and $\mathbf{b}$ Orthogonal curved MIP views of the right coronary artery. Because of the large amount of calcified plaque, the vessel lumen is not visualized on curved MIP projections. cCTA was not able to clear this vessel, even though conventional cardiac catheterization did not demonstrate an area of high-grade stenosis increased above $20 \%$, cCTA alone may be a more appropriate option for the diagnostic workup in order to avoid a large number of false-negative test results that are associated with stress testing [9]. Based upon the high negative predictive value, ready availability and rapid diagnostic time of cCTA for the exclusion of coronary disease, cCTA has been proved to be a cost-effective examination for evaluation of low to intermediate risk patients with suspected acute coronary syndrome presenting to the emergency department $[11,12]$.

cCTA examinations are usually performed as a dedicated study of the coronary arteries, tailored to image the coronary arteries with a minimum dose of iodinated contrast and radiation exposure. However, in the setting of a patient who presents with acute chest pain, the differential diagnosis often includes other potentially life-threatening processes such as pulmonary embolism and aortic dissection. A dedicated cCTA will not include the aortic arch. Furthermore, contrast injection for a dedicated cCTA is usually timed with a saline bolus to wash out the right heart during imaging. In order to optimize imaging of the coronary arteries, thoracic aorta and pulmonary arteries, a triple ruleout protocol may be applied [13]. When performed with proper attention to technique, the triple rule-out study provides excellent opacification of the aorta and pulmonary arteries without loss of coronary artery image quality, 

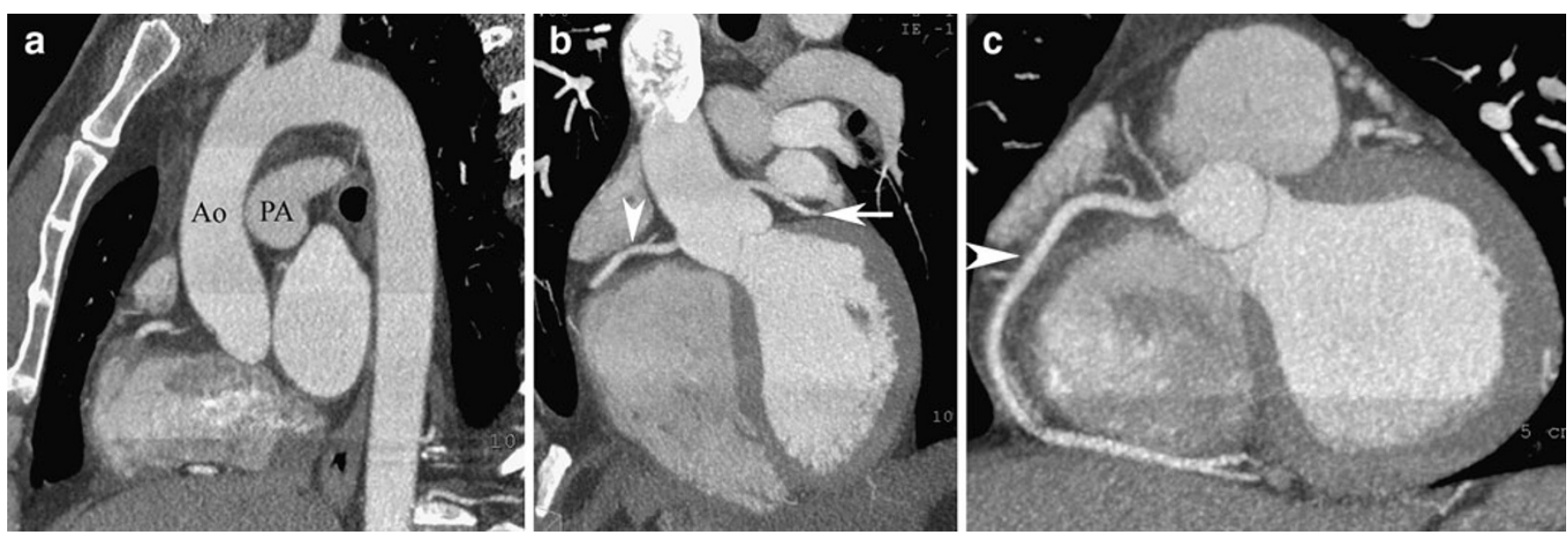

Fig. 7 Triple rule-out study with opacification of the aorta, coronary arteries and pulmonary arteries. a Oblique sagittal MIP demonstrates the thoracic aorta (Ao) as well as the bifurcation of the pulmonary arteries (PA). This scan was acquired in four steps using prospective ECG-gating to reduce patient dose. A linear artifact is apparent between each step. b Left anterior oblique coronal MIP demonstrates

although there is an increased radiation dose proportional to the increased scan length (Fig. 7) [14].

cCTA is currently not recommended for evaluation of asymptomatic patients [15]. Atherosclerotic CAD is highly prevalent in the population of developed countries, and screening for CAD by cCTA is likely to identify many patients with $\mathrm{CAD}$ who might not benefit from interventional therapy. Several recent studies have suggested that cCTA may be useful for risk assessment in the asymptomatic population (see below), but this suggestion must be tempered by the potential for overtreatment of anatomical disease, which is found during such "screening cCTA" procedures. A similar problematic situation arises when low-risk asymptomatic patients are referred for cardiac catheterization after a positive stress test in the course of a

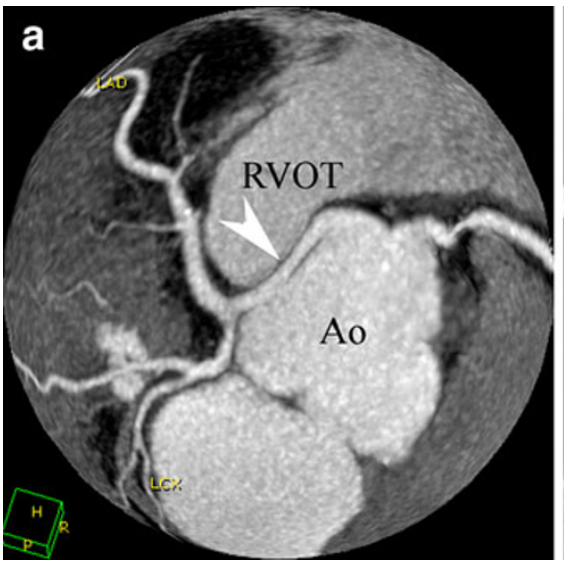

Fig. 8 Anomalous left coronary artery with a "malignant" course between the aorta (Ao) and the right ventricular outflow tract (RVOT). a Globe MIP demonstrates the course of the left coronary artery (arrowhead), which originates from the right sinus of Valsalva and travels between the aorta (Ao) and right ventricular outflow tract the origins of the right coronary artery (arrowhead) and left coronary artery (arrow). The aortic valve is identified between the left ventricle and the ascending aorta. c Slightly steeper left anterior oblique projection demonstrates the classic " $\mathrm{C}$ " shape of the right coronary artery

preoperative or "routine" cardiac evaluation. Such patients may benefit from a "second opinion" cCTA, which may obviate the performance of an unnecessary invasive conventional diagnostic cardiac catheterization [16].

\section{Calcium scoring and cCTA for risk assessment}

An overwhelming majority of patients presenting with acute coronary syndrome have calcification in the coronary circulation. Several large retrospective studies of asymptomatic patients have demonstrated that the presence of coronary calcium is a predictor of cardiac events [17] and risk of death $[18,19]$. Prospective studies have confirmed these results and demonstrated that coronary calcium scoring provides

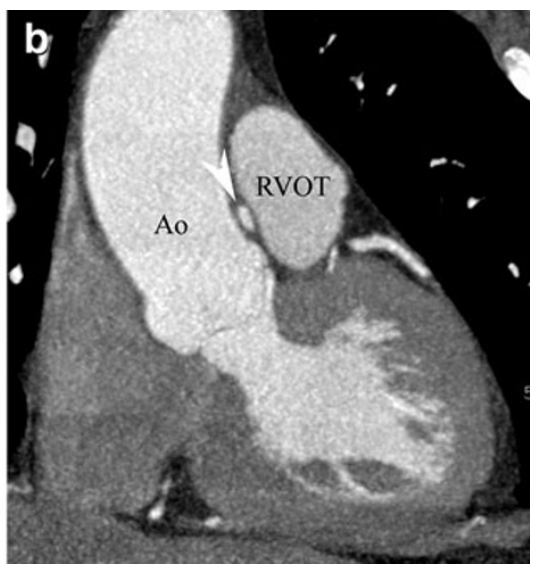

(RVOT). The left coronary artery is seen to branch into the left anterior descending (LAD) and circumflex (LCX) arteries. b Sagittal MIP demonstrates compression of the left coronary artery (arrowhead) into an ovoid shape as it courses between the aorta (Ao) and right ventricular outflow tract (RVOT) 

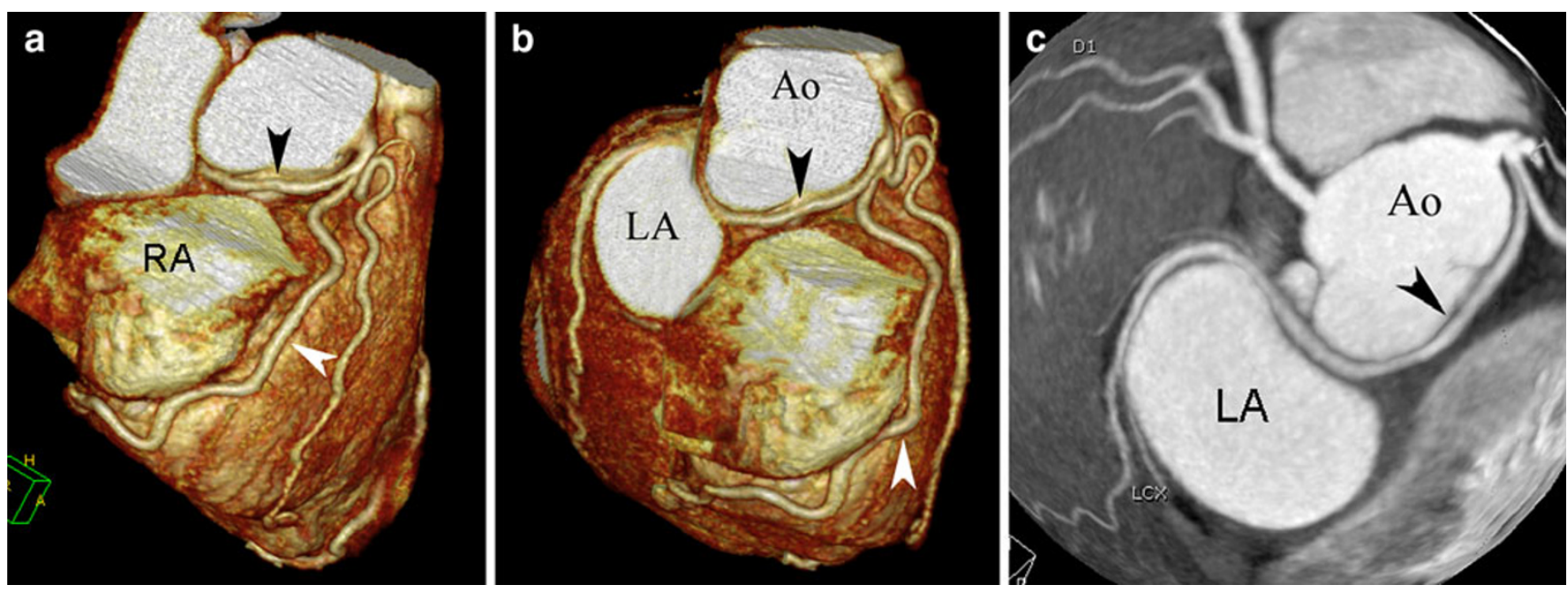

Fig. 9 Anomalous origin of the circumflex artery from the right coronary artery with a "benign" course posterior to the aorta. a Volumetric-rendering demonstrates the right coronary artery (white arrowhead) as it courses in the right atrioventricular groove. The circumflex artery (black arrowhead) branches off the right coronary artery and courses posterior to the aorta. b Volumetric rendering from

additional prognostic information beyond standard risk assessment based upon clinical and laboratory tests [20, 21]. Nonetheless, calcium scores are not recommended to evaluate the patient who presents with acute symptoms. Coronary calcium imaging does not detect the presence of non-calcified plaque, and a minority of patients experience coronary events in the absence of coronary calcification (Fig. 2) [22, 23].

A recent meta-analysis of 64-slice MDCT studies included 10 studies with 5,675 patients. After a mean follow-up of 21 months, major adverse cardiac events occurred in $0.5 \%$ of patients with normal cCTA, $3.5 \%$ of patients with nonobstructive disease on cCTA and $16 \%$ of patients with obstructive cCTA $(p=0.0001)$ [24]. Individual studies have demonstrated an increasing frequency of cardiac events based upon both increasing plaque burden and more proximal location [25]. Although cCTA is not currently recommended for risk stratification, continued advances in plaque characterization by CTA may make risk stratification by cCTA a reality in the future [26].

\section{CT perfusion imaging}

A major limitation of traditional cCTA is the lack of a functional imaging component to gauge whether the coronary stenosis results in myocardial ischemia. The extent of inducible ischemia associated with coronary disease is predictive of the risk of a coronary event as well as the expected benefit of revascularization compared with medical therapy $[27,28]$. In order for cCTA to serve as a comprehensive evaluation for coronary disease, the study a more superior projection again demonstrates the right coronary artery (white arrowhead) as it courses in the right atrioventricular groove. The circumflex artery (black arrowhead) courses between the aorta (Ao) and left atrium (LA). c Globe MIP again demonstrates the circumflex artery (black arrowhead), which courses between the aorta (Ao) and left atrium (LA)

needs to provide both anatomic and functional information about CAD.

Several groups have used CT to perform perfusion imaging either as a stand-alone examination or as part of a cCTA study. A first-pass dynamic evaluation of myocardial perfusion might be the most accurate technique to quantify myocardial perfusion, but such an approach requires repeated scanning of the heart with a relatively high radiation exposure. Several groups have used a single arterial phase to estimate myocardial perfusion based upon myocardial blood pool enhancement during CCTA and a delayed phase to image late enhancement that may be associated with infarcted myocardium [29]. Low-dose scans for late enhancement can provide valuable diagnostic information on the functional significance of coronary artery disease [30].

Given the relatively small changes in CT density provided by enhancement of the myocardium with iodinated contrast material, various techniques have been proposed to improve the sensitivity of CT for perfusion imaging. One group has suggested that ischemic myocardium may be recognized by a pattern of subendocardial hypoperfusion during systole with apparent normal perfusion during diastole [31]. Using this technique, they have demonstrated a sensitivity of $90 \%$ and specificity of $83 \%$ for CT detection of myocardial perfusion defects identified by nuclear perfusion imaging [32].

A more conventional approach to identify ischemic myocardium with CTA uses pharmacologic stress. Given the limited temporal resolution of $\mathrm{CT}$, the tachycardia associated with a dobutamine stress study can result is substantial degradation of image quality. However, adeno- 
sine may be used as a vasodilator for CT stress imaging. One group has measured a transmural perfusion ratio during adenosine stress CT (subendocardial attenuation density/ subepicardial attenuation density) to detect myocardial perfusion abnormalities with a sensitivity/specificity of $86 \% / 92 \%$ on a per-patient analysis [33]. Another group has combined adenosine with first pass stress imaging followed by rest images, and has demonstrated good correlation with SPECT myocardial perfusion imaging for both stress and rest imaging [34, 35]. The combination of cCTA stress imaging with subsequent rest imaging as well as a third scan for delayed enhancement can be performed with an effective radiation exposure similar to that of SPECT imaging (mean $12.7 \mathrm{mSv}$ ) [35].

In order to improve the detection of perfusion defects with cCTA, dual-energy CT may be useful. A relatively higher $\mathrm{kVp}$ is needed for optimal tissue penetration to optimize coronary imaging, while a lower $\mathrm{kVp}$ is useful to improve sensitivity for the detection of small differences in myocardial enhancement. A dual-source scanner may be used to simultaneously provide adequate penetration for coronary cCTA as well as low $\mathrm{kVp}$ imaging to improve detection of myocardial perfusion. A low energy "iodine perfusion map" is displayed as a color overlay on the high-resolution
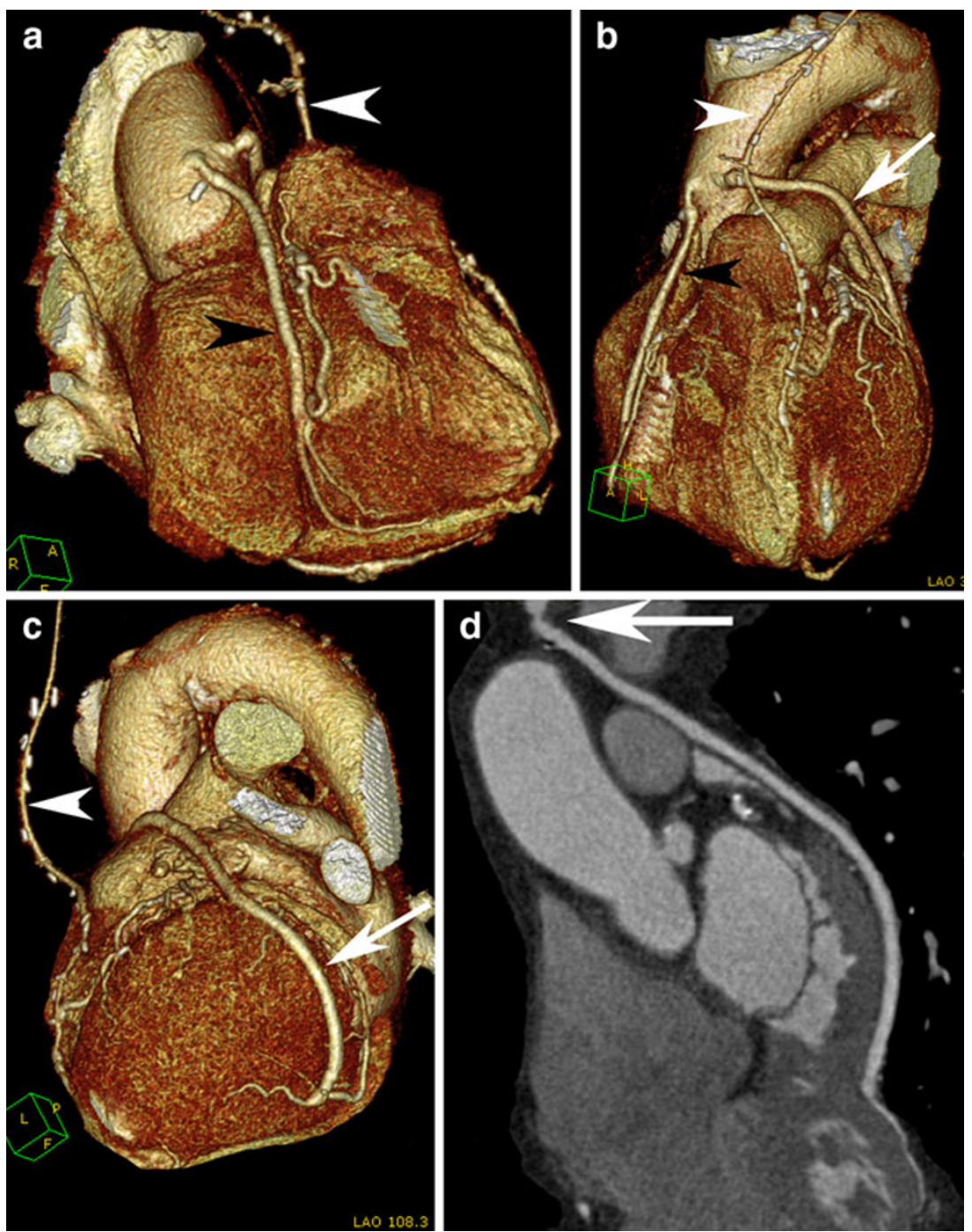

Fig. 10 Patent bypass grafts with narrowing at the origin of the vein graft to the circumflex distribution. a Volumetric-rendering in the right anterior oblique demonstrates a vein graft (black arrowhead) coursing from the aorta to the distal right coronary artery. The superior portion of the left internal mammary artery (LIMA) bypass is also visible (white arrowhead). b Volumetric rendering in the left anterior oblique again demonstrates the vein graft (black arrowhead) from the aorta to the distal right coronary artery. The left internal mammary artery (LIMA) bypass (white arrowhead) is seen to course into the anterior interventricular groove where is anastamoses with the left anterior descending artery. A second vein graft (arrow) extends from the aorta to the circumflex territory. c Volumetric rendering in the left lateral projection viewed from a more inferior approach again demonstrates the left internal mammary artery (LIMA) bypass (white arrowhead) to its anastamosis. The anastamosis of a vein graft (arrow) to an obtuse marginal branch of the circumflex artery is visualized. d Curved MIP of the vein bypass to the circumflex artery territory. There is greater than $50 \%$ narrowing at the origin of this vein graft (arrow) 


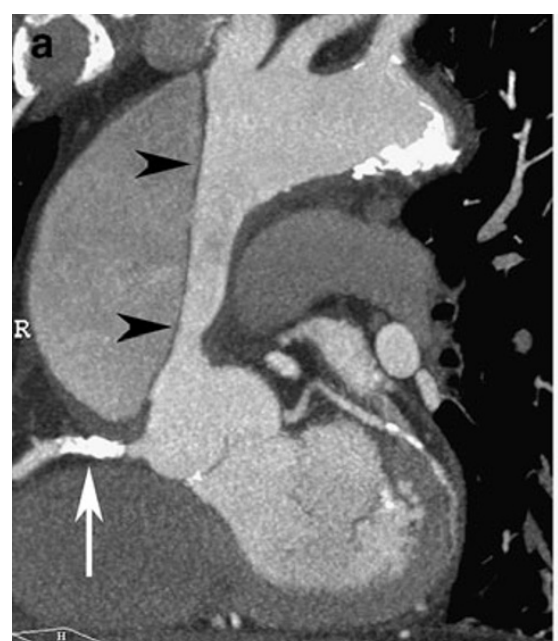

Fig. 11 Type A dissection of the ascending aorta with extension into the aortic root. a Oblique MIP demonstrates a dissection flap in the ascending aorta (black arrowheads). The dissection extends down to the right coronary artery (white arrow). A metallic stent is present in the proximal right coronary artery, obscuring the underlying vessel. b

coronary CTA image to provide excellent diagnostic correlation with SPECT imaging (sensitivity: 91\%, specificity: 92\%) [36]. This same group demonstrated cCTA detection of reversible SPECT defects without stress imaging [37]. They have suggested that such defects may be visible without stress imaging because of the superior spatial resolution and dynamic range of $\mathrm{CT}$ as compared with nuclear SPECT or secondary to the intrinsic vasodilatory effects of iodinated contrast [38].

\section{Additional applications of cCTA}

cCTA provides clear definition of the anatomic course of the coronary arteries, even when image quality is limited with respect to assessment of coronary stenosis. Although the coronary arteries are clearly visualized by conventional cardiac catheterization, cCTA more clearly demonstrates the relationship between coronary arteries and adjacent anatom-

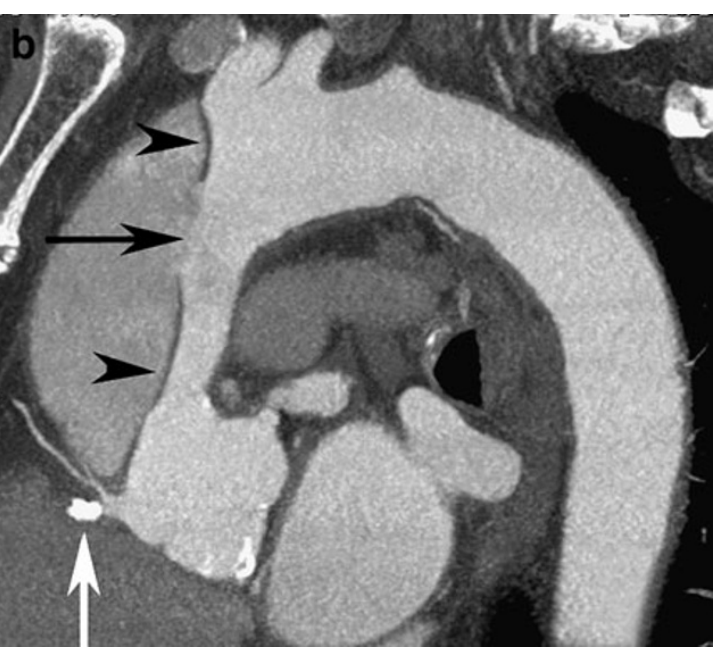

Oblique MIP again demonstrates a dissection flap in the ascending aorta (black arrowheads). The communication between the true lumen and false lumen is visualized as a defect in the dissection flap (black arrow). The proximal right coronary artery is again visualized (white arrow)

ical structures such as the pulmonary artery. For this reason, cCTA is an excellent study to demonstrate various coronary anomalies (Figs. 8, 9). cCTA is often superior to conventional arteriography for the diagnosis of coronary variations [39].

cCTA provides a useful roadmap in preoperative planning for bypass surgery as well as in post-surgical assessment. In addition to localizing the site of coronary stenosis, cCTA can be used to assess whether a more distal segment of a coronary vessel is epicardial in location and is accessible for bypass grafting [40]. In the postoperative patient, cCTA can be used to assess the patency of bypass grafts that may be difficult to cannulate by conventional arteriography (Fig. 10). Injury to existing bypass grafts is a major risk of reoperation. cCTA clearly demonstrates the position of bypass grafts relative to other structures and is therefore useful for planning of reoperation bypass procedures [41].

The stable patient with a suspected thoracic aortic injury, dissection or aneurysm is often evaluated with a CT for preoperative planning as well as a cardiac catheterization to

Fig. 12 a and b Status post repair of type A dissection with a mechanical aortic valve (white arrowhead) at the aortic annulus and a tube graft (white arrow) of the ascending aorta above the native aortic root. A dissection flap is present beyond the tube graft (black arrowheads) with visible communication between the true lumen and false lumen
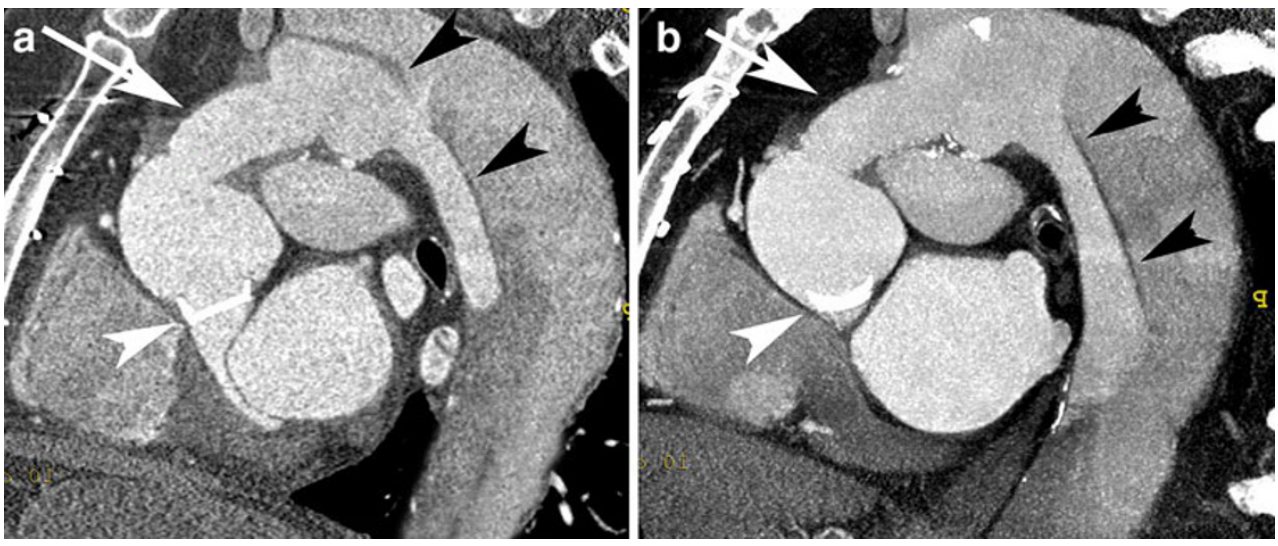

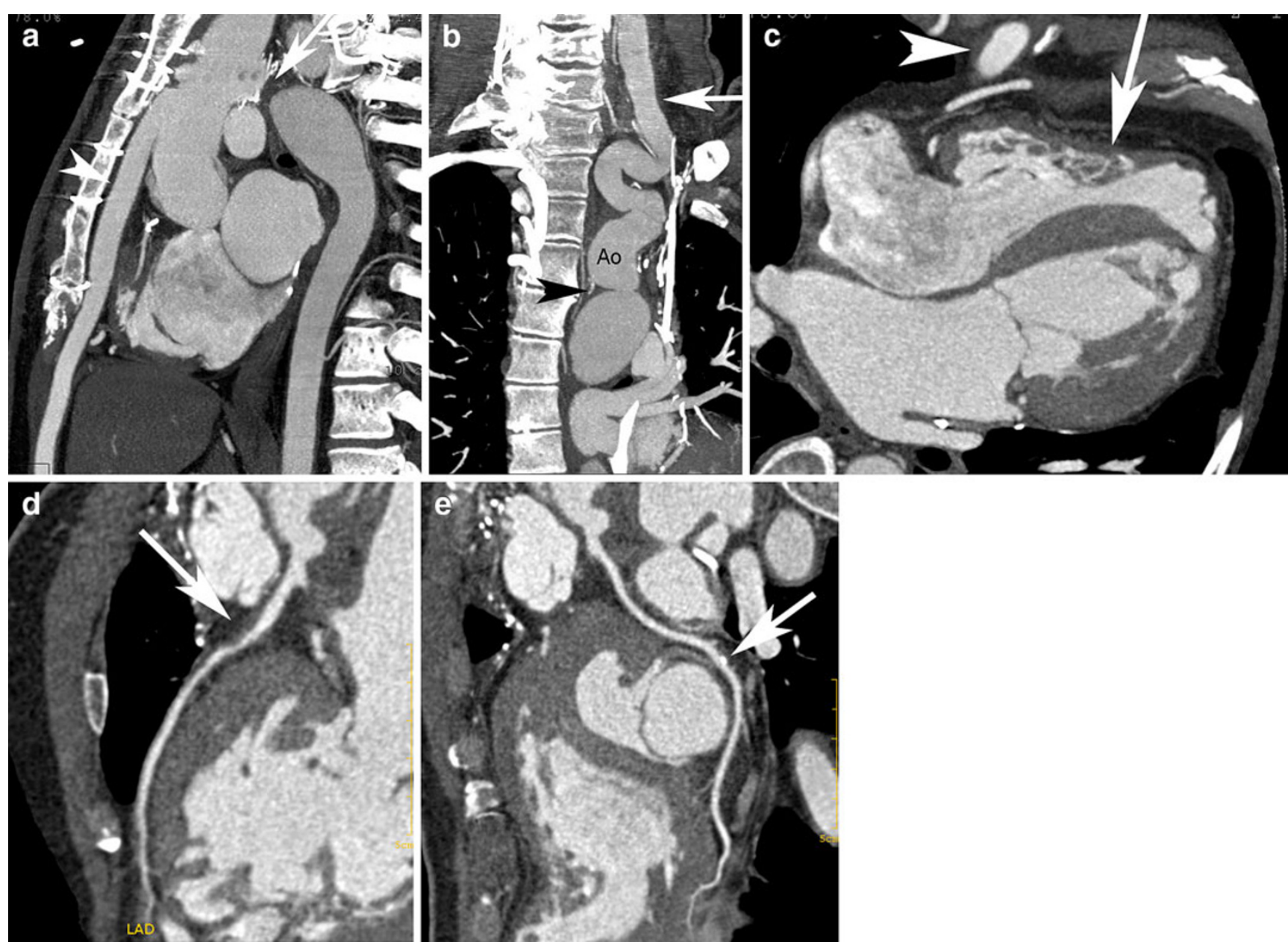

Fig. 13 Interruption of the aortic arch with collateral circulation and graft. Mild coronary disease is identified without a hemodynamically significant lesion. a Sagittal MIP demonstrates the interruption (arrow) with a large left common carotid exiting the aortic arch just proximal to the interruption. There is decreased opacification of the distal thoracic aorta relative to the ascending aorta. An aorto-femoral graft extends caudally from the ascending aorta and is visualized in the retrosternal space (arrowhead). b Coronal MIP demonstrates a large, tortuous collateral branch (arrow) extending down from the left external carotid artery to the aortic arch, just beyond the interruption.
A mild coarctation is visible in the proximal descending aorta (black arrowhead). This collateral branch and the coarctation were not visible in the sagittal MIP. $\mathbf{c}$ Four-chamber view of the heart demonstrates the aorto-femoral graft anteriorly (arrowhead). There is an unusual contour to the right ventricle, which appears to be narrowed in its mid-portion (arrow). d Curved MIP view of the LAD demonstrates non-calcified plaque in the proximal portion of this vessel with mildmoderate - approximately 50\% - narrowing (arrow). e Curved MIP view of the LCX demonstrates eccentric calcified plaque in the mid portion of this vessel without narrowing (arrow)
Fig. 14 Normal aortic valve in short axis. a Closed aortic valve demonstrates normal coaptation of the leaflets in diastole at $70 \%$ of the R-R interval. Three raphe lines are visible within the valve (arrowheads). b Aortic valve opens with a triangular shape, demonstrating three independent leaflets at $30 \%$ of the R-R interval. The presence of a trileaflet valve can only be confirmed by demonstrating this normal systolic opening
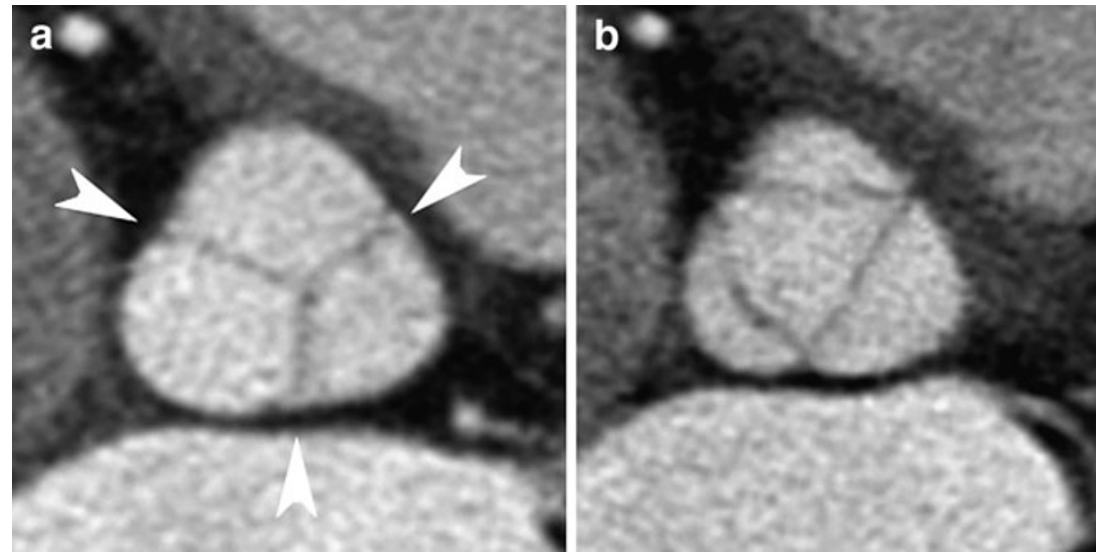
Fig. 15 Mildly thickened aortic valve with normal motion pattern. a Closed aortic valve demonstrates normal coaptation of the leaflets at the three raphe lines (arrowheads). b Systolic image demonstrates a normalsize opening with mild myxomatous thickening and redundancy of the leaflets
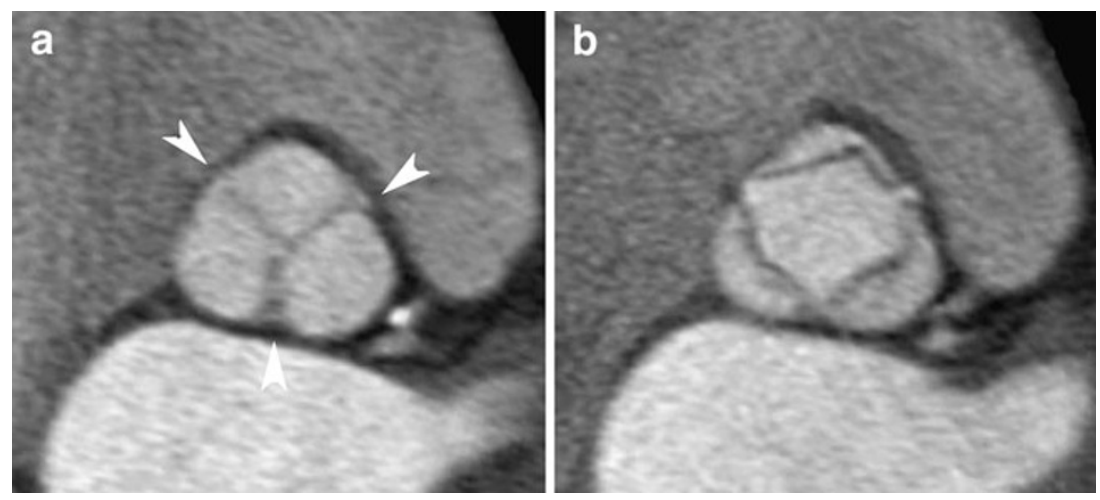

assess patency of the coronary arteries. cCTA can provide simultaneous evaluation of pathology in the aorta as well as assessment of the coronary arteries for preoperative planning (Fig. 11). Following repair of an aortic aneurysm, cCTA can provide clear anatomic definition of prosthetic valves, aortic grafts and residual dissection flaps (Fig. 12). cCTA is also useful for evaluation of complex aortic anomalies such as coarctation, interruption of the aortic arch and collateral vascular supply (Fig. 13).

Preoperative planning for aortic valve replacement surgery requires assessment of the ascending aorta for morphology and calcification, assessment of the aortic valve annulus for appropriate sizing of the replacement valve and evaluation of the coronary arteries. cCTA is an excellent modality to assess simultaneously the thoracic aorta, the aortic valve and the coronary arteries (Figs. 14, 15). cCTA can provide accurate imaging and measurements of the aortic annulus and valve for preoperative planning of aortic valve replacement [42]. Percutaneous valve replacement procedures are poised to revolutionize the therapy of aortic valve disease [43]. cCTA provides essential information prior to percutaneous valve replacement, especially with respect to the location and extent of aortic root and valve calcifications and the location of the coronary ostia relative to the valve plane [44]. The diagnosis of bicuspid valve is readily apparent on cCTA (Fig. 16), and the presence of aortic valve thickening and insufficiency may also be visible (Fig. 17).

When reoperation is required for a prosthetic aortic valve that has failed, coronary angiography is often contraindicated, especially in the setting of endocarditis when catheter angiography carries an increased risk of embolization [45]. cCTA can provide high-resolution functional imaging of prosthetic valves with simultaneous evaluation of the coronary arteries for preoperative planning. Prosthetic endocarditis may also be associated with additional complications including valve malfunction and pseudoaneurysms, which may be clearly defined by cCTA (Fig. 18).

MRI is generally considered the test of choice for evaluation of cardiac morphology since this technique does not require ionizing radiation. However, cCTA has been used extensively in preoperative planning for ablation procedures in the left atrium. The pulmonary veins and the intricate details of the left atrial anatomy are more clearly visualized by cCTA, which provides superior spatial resolution as compared to MRI, and their location can be mapped into a

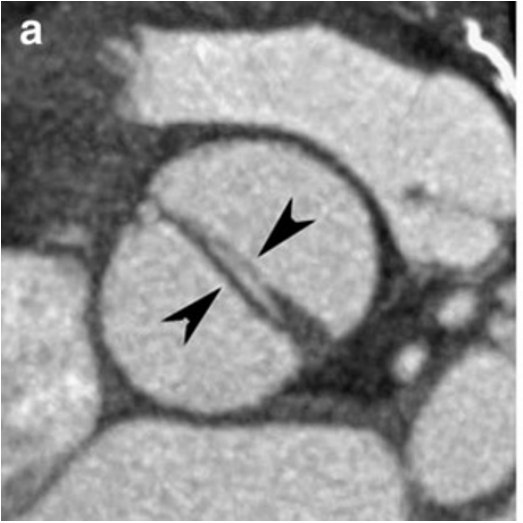

Fig. 16 Bicuspid aortic valve with regurgitation and minimal restriction of motion. a Diastolic image in short axis of the aortic valve demonstrates a linear closure of the aortic valve with only two leaflets (arrowheads). A small regurgitant orifice is visible between the mildly thick leaflets. The more anterior leaflet is in the position that

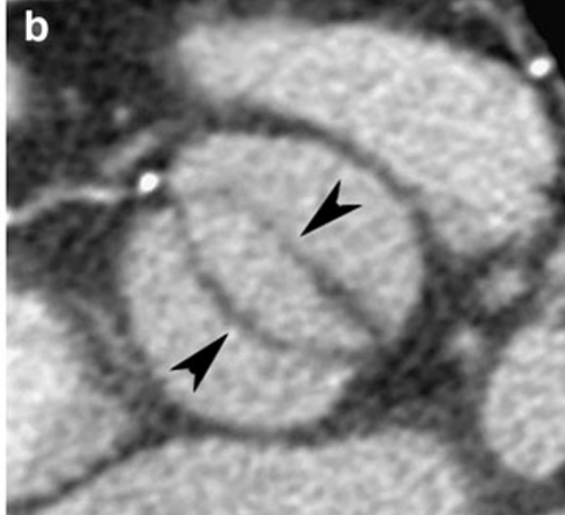

would typically be occupied by the right and left coronary leaflets. b Systolic image in short axis of the aortic valve demonstrates a "fishmouth"-shaped opening of the two leaflets (arrowheads) that is typical for a bicuspid valve. The overall aortic valve area is minimally restricted $\left(2 \mathrm{~cm}^{2}\right.$ by planimetry) 

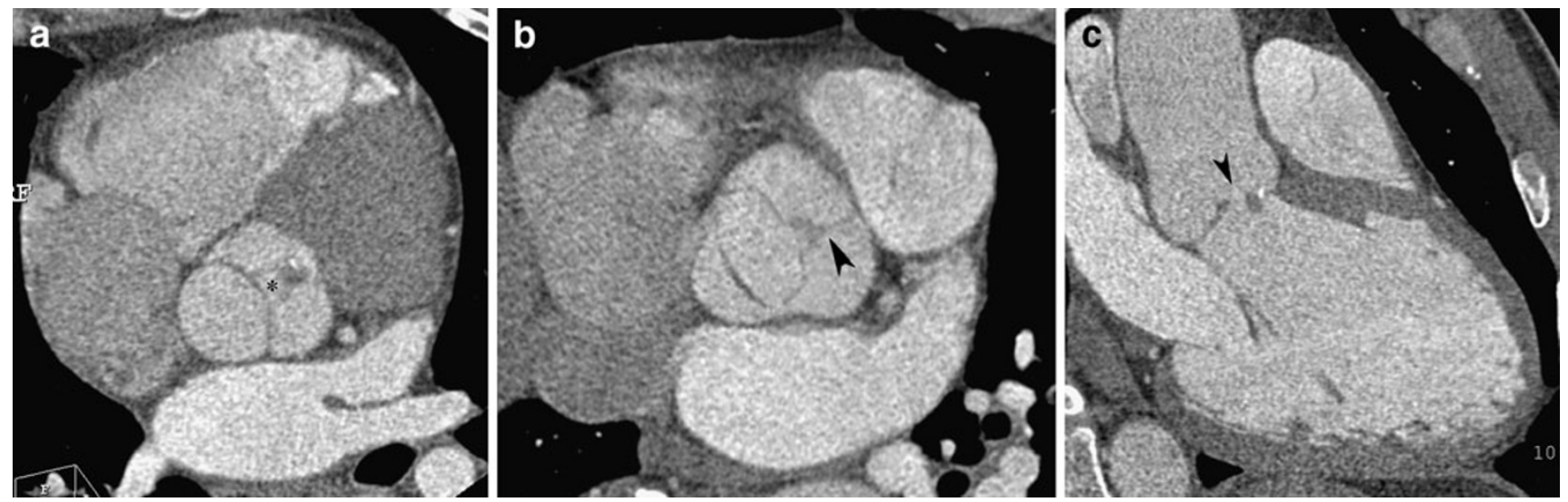

Fig. 17 Bicurpid aortic valve with moderate aortic insufficiency and focal thickening, likely related to endocarditis. a Diastolic image in short axis of the aortic valve demonstrates incomplete coaptation of the valve leaflets with a regurgitant orifice $(*)$. Just anterior to this regurgitant orifice, there is a focal thickening of the valve, representing a vegetation. b Systolic image in short axis demonstrates a "fishmouth" opening of the aortic valve with a visible raphe (arrowhead) within the anterior leaflet. This raphe is actually a "pseudoraphe" as it does not open as a normal raphe, but can mimic the appearance of a trileaflet aortic valve on diastolic imaging. The regurgitant orifice in diastole appears to be related to the free edge of this pseudoraphae. c Three-chamber view in diastole demonstrates incomplete coaptation of the aortic valve (black arrowhead), with focal thickening (a vegetation) associated with the free edge of the anterior leaflet three-dimensional volume that is used during the ablation procedure (Fig. 19). Integration of CTA image data during ablation in the left atrium is often useful to shorten procedure times and improve patient outcome [46]. Although echocardiography and MRI are generally preferred for evaluation of cardiac morphology, changes in cardiac morphology (Fig. 20) and the presence of mass lesions (Fig. 21) can be evaluated by cCTA.

Enlargement of the right heart is often related to intracardiac shunts. cCTA is an excellent test for identification of anomalous pulmonary veins that are not visualized by echocardiography. Furthermore, we have found cCTA to be a useful study in the pre-procedure planning for percutaneous closure of atrial septal defects (Fig. 22) and ventricular septal defects (Fig. 23). The superb three-dimensional anatomical definition provided by $\mathrm{CT}$ imaging allows assessment of the size and location of the defect relative to adjacent structures such as the aortic root.

Evaluation of the right heart by cCTA is limited by the mixing of opacified blood from the SVC with unopacified blood from the IVC. Nonetheless, with appropriate modifications of the contrast injection protocol, cCTA may be used successfully to evaluate right ventricular morphology, as well as for evaluation of the tricuspid valve (Fig. 24) [14].

Given the development of new low-dose CTA protocols (see below) and the more rapid acquisition time of CTA

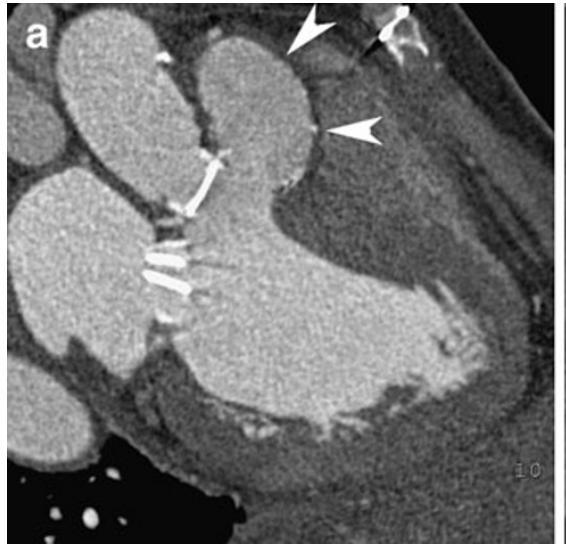

Fig. 18 Pseudoaneurysm of the left ventricular outflow tract following prosthetic valve endocarditis. a Three-chamber view during diastole demonstrates an open St. Jude's valve in the mitral position and a closed St. Jude's valve in the aortic position. A pocket of contrast just anterior to the aortic prosthesis (arrowheads) represents a

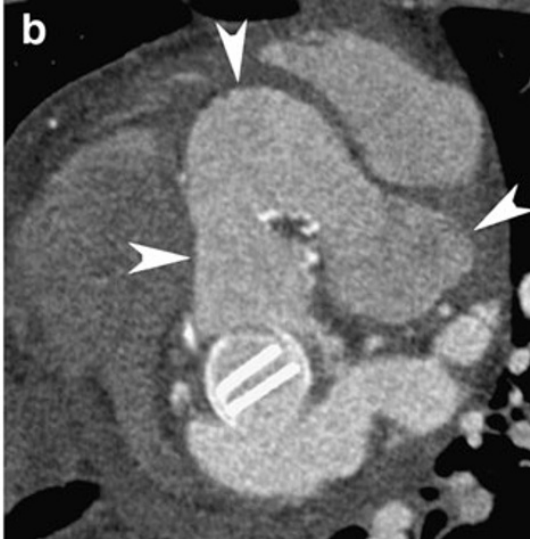

pseudoaneurysm of the left ventricular outflow tract. b Axial image during systole demonstrates an open aortic prosthesis in short axis. The pseudoaneurysm is visualized to extend as a tubular structure into the aorto-pulmonary window (arrowheads) 

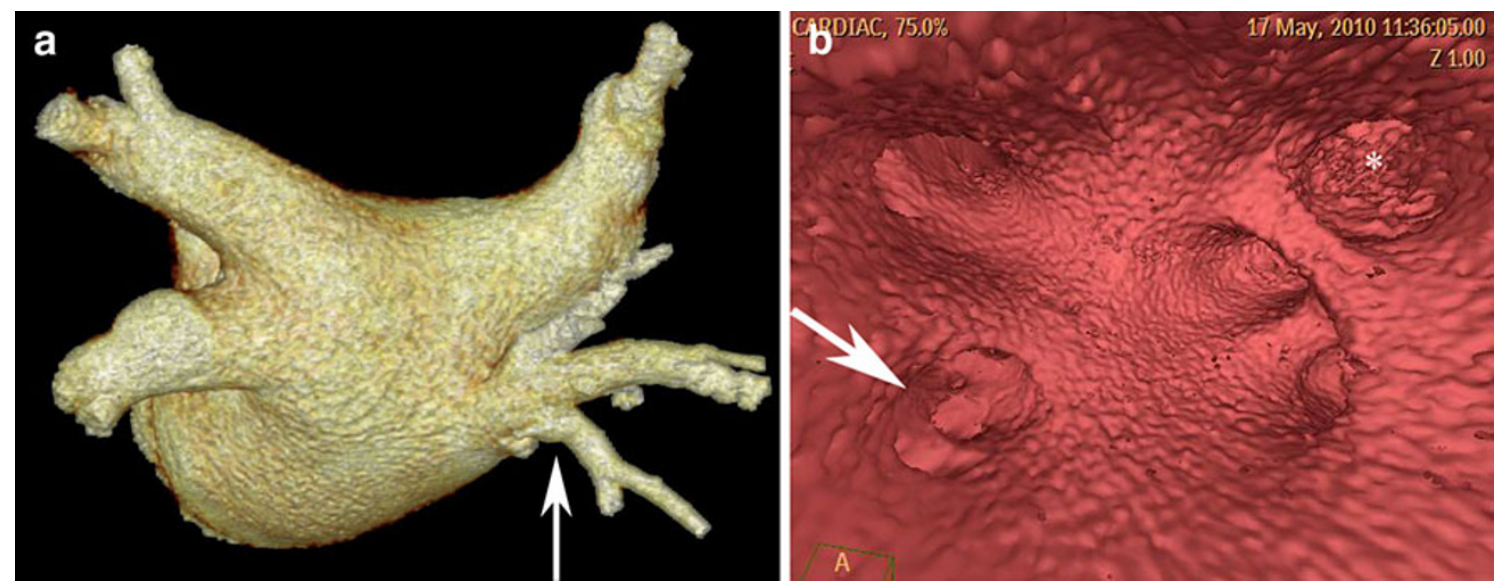

Fig. 19 Pulmonary venous mapping of the left atrium demonstrates a compound right inferior pulmonary vein. a Volumetric surface rendering of the left atrium from a posterior vantage point demonstrates four pulmonary veins. The right inferior vein consists of several small branches that unite at the level of the left atrium (arrow). This information is important when performing an ablation procedure,

as these branches have small orifices that might be damaged with resulting pulmonary vein stenosis. b Endoluminal rendering demonstrates multiple small branches entering the left atrium in the location of the right inferior pulmonary vein (arrow). The orifice of the left atrial appendage $(*)$ is separated from the two left-sided pulmonary veins by the warfarin ridge
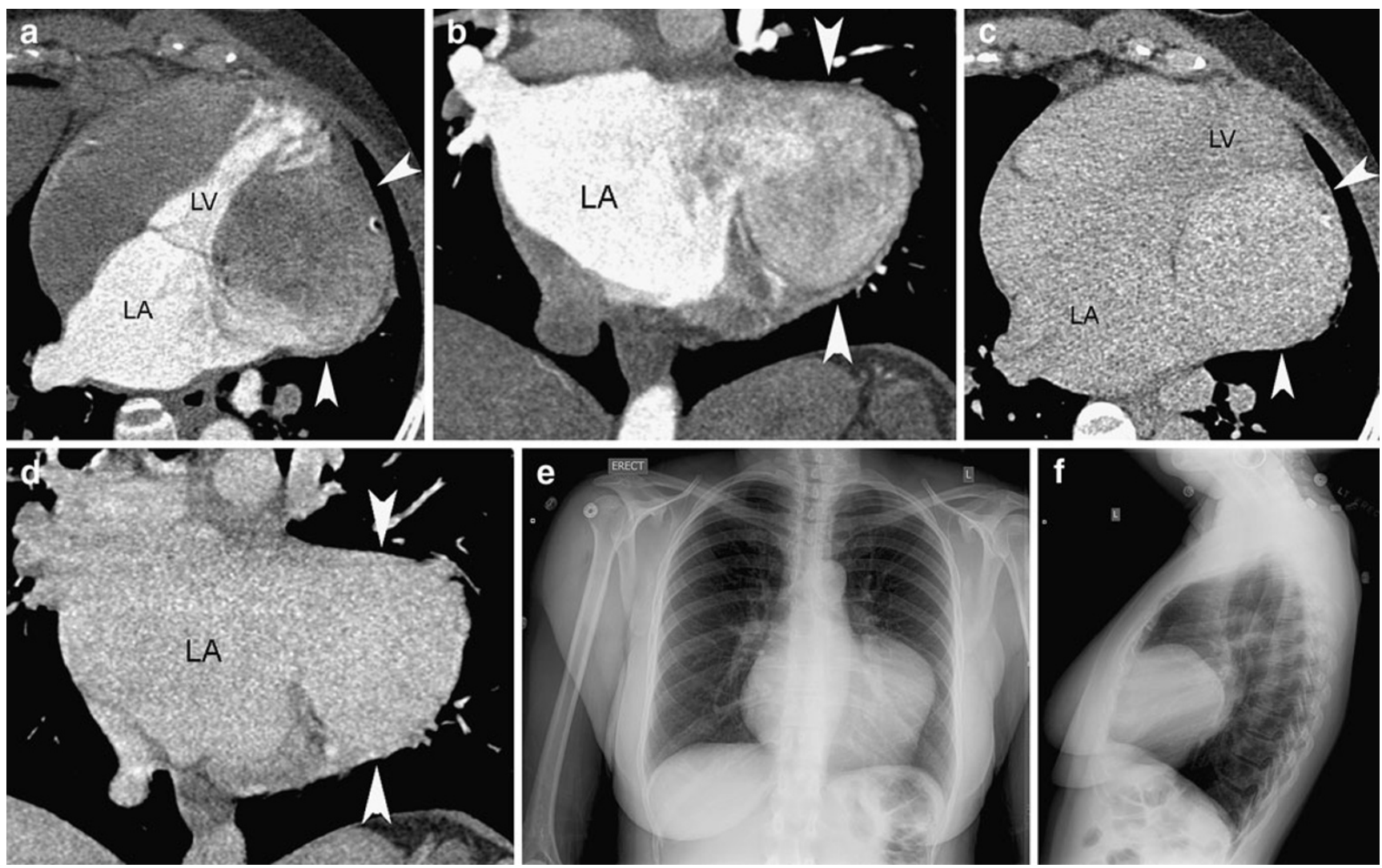

Fig. 20 Partial absence of the pericardium with herniation of the left atrial appendage. a Four-chamber view of the heart demonstrates a mass along the left heart border (arrowheads) adjacent to the left atrium (LA) and left ventricle (LV). A small amount of contrast material enters this mass. b Coronal view of the left atrium (LA) demonstrates that this mass (arrowheads) corresponds to the typical location of the left atrial applendage. Stagnant flow within the mass results in slow filling, but no thrombus was visible on delayed views. c Four-chamber view in a delayed phase demonstrates contrast filling of the mass. d Coronal view in a delayed phase also demonstrates homogeneous enhancement of the mass that represents a herniated and dilated left atrial appendage. e PA view of the chest demonstrates a bulge in the left cardiac contour. $\mathbf{f}$ Lateral view of the chest again demonstrates the mass corresponding to the herniated and enlarged left atrial appendage 
Fig. 21 Left atrial myxoma in a patient with a recent transient ischemic episode. a Axial image demonstrates a small focal mass within the left atrium (arrowhead). The more common location for a left atrial appendage would be along the interatrial septum. b Sagittal image demonstrates that this mass (arrowhead) extends from the roof of the left atrium adjacent to the orifice of the left atrial appendage
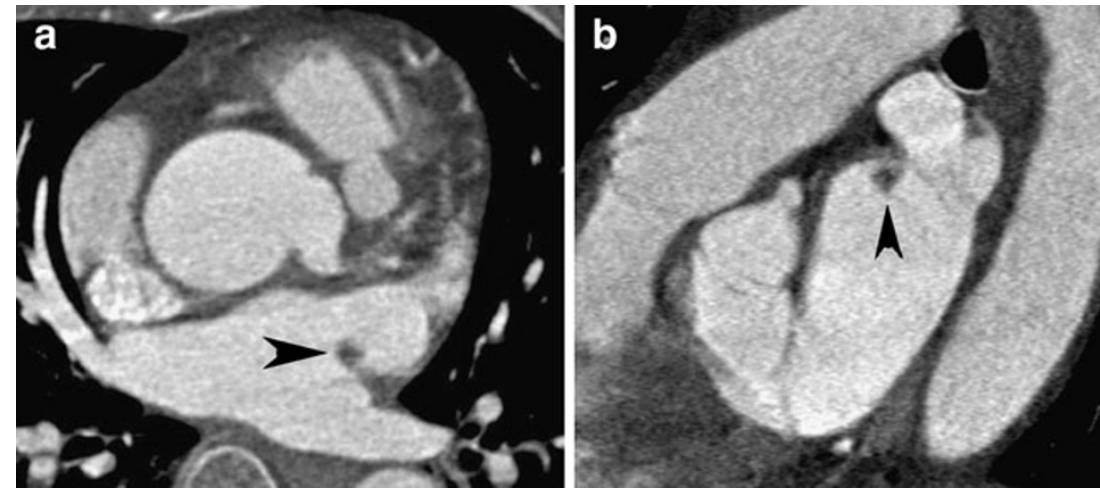

compared to MRI, it is likely that cCTA will play an increasing role in the evaluation of cardiac morphology and congenital heart disease. As a full discussion of these topics is beyond the scope of this pictoral review, the reader is referred to a recent textbook for more in-depth discussion of these topics [47].

\section{Limitations of cCTA/alternative studies}

cCTA is a powerful diagnostic tool, but necessitates injection of intravenous contrast material as well as exposure to ionizing radiation. cCTA is often limited by image quality in obese patients as well as patients with elevated or irregular heart rates. The presence of blooming artifact from dense arterial calcification limits the quality of cCTA and results in overestimation of the degree of stenosis (Fig. 6). Evaluation of metallic stents is also limited by blooming artifacts from the stent struts, though cCTA has been increasingly successful for evaluation of coronary stents, especially with stent diameters above $3 \mathrm{~mm}$ (Figs. 25, 26). Although a normal cCTA effectively excludes the presence of CAD, the finding of moderate disease by cCTA is often of uncertain clinical significance as it may not be obvious whether the patient's symptoms are related to the visualized CAD. Because of these limitations, competing technologies for evaluation of suspected CAD - including ECG-stress testing, stress testing with echocardiography, stress testing with nuclear perfusion imaging and MRI-may be more appropriate in specific clinical scenarios. Functional stress testing may be appropriate to evaluate the clinical significance of CAD detected by cCTA.

A meta analysis of 147 consecutively published reports of exercise treadmill studies reported a sensitivity of $68 \%$ with a specificity of $77 \%$ for the detection of CAD [48]. Diagnostic accuracy is somewhat higher for stress echocardiography and stress perfusion imaging, with both of these tests demonstrating superior diagnostic accuracy as com-

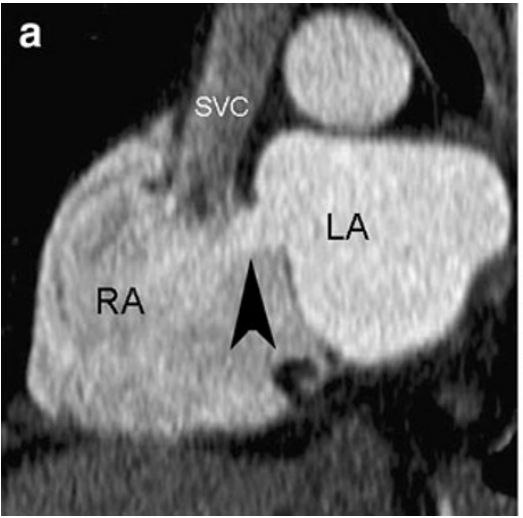

Fig. 22 Atrial septal defect (ASD) with left to right shunt. a Sagittal MIP demonstrates a posterior left atrium (LA), with a more anterior right atrium (RA). The superior vena cava (SVC) is identified as it enters the right atrium. A jet of contrast material (arrowhead) extends from the left atrium through an ASD into the right atrium, just below the orifice of the SVC. The proximity of the ASD to the SVC is clearly demonstrated. b Axial MIP demonstrates the ASD with left to

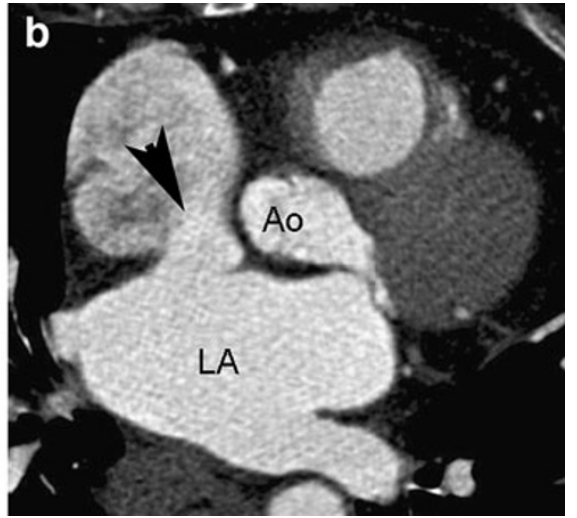

right shunting of contrast (arrowhead). The proximity of the ASD to the aortic root (Ao) is clearly demonstrated. The location of the ASD and the presence of a rim of tissue must be ascertained prior to attempted percutaneous closure. If the ASD is too close to the aortic root, the closure device may clamp the root with a possible long-term complication of erosion into the aortic root 
Fig. 23 Post-infarct ventricular septal defect. a Four-chamber view demonstrates a defect in the distal portion of the interventricular septum (arrowhead). Trabeculations within the right ventricle overlie the defect. b Short axis view demonstrates that the defect is larger than appreciated on the four-chamber view and extends through the inferior half of the distal septum (arrowhead)
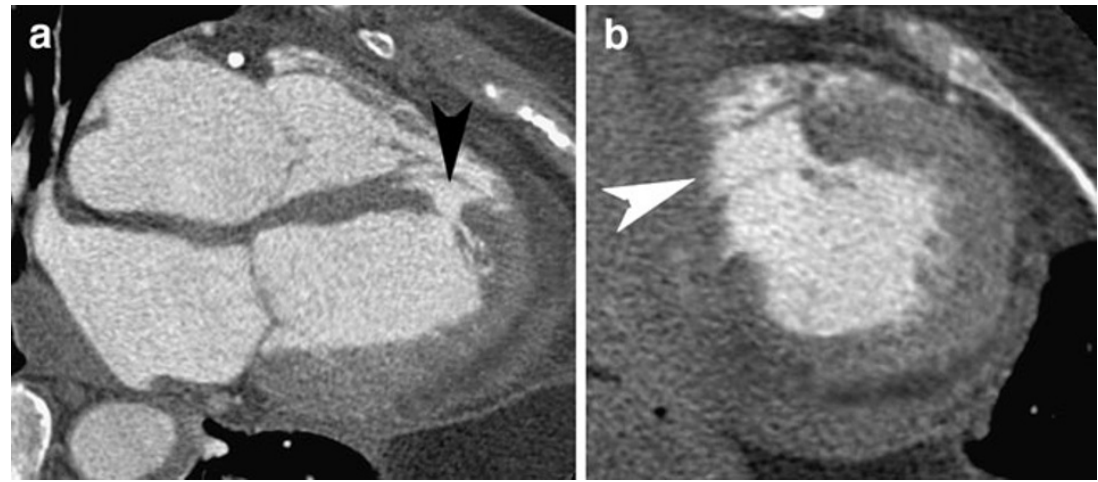

pared to the basic ECG stress treadmill test. A pooled analysis of stress tests for the diagnosis of "significant" CAD demonstrated a sensitivity/specificity of $80 \% / 86 \%$ for stress echocardiography and $84 \% / 77 \%$ for stress perfusion imaging [49]. Although most studies suggest a relatively similar diagnostic accuracy for stress echocardiography and stress perfusion imaging, a recent meta-analysis suggests that stress echocardiography is superior to stress perfusion imaging for evaluation of high risk (left main disease and/or triple vessel) disease because of the greater risk of missing severe CAD with perfusion imaging (sensitivity $75 \%$ versus 94\%) [50]. Although echocardiography does not provide direct visualization of the coronary arteries, stress ECG combined with echocardiography is a well-established noninvasive examination for stratifying the risk of CAD and influencing the decision for subsequent coronary angiography and revascularization [51].

Based upon the decision analysis cited above, initial evaluation of suspected CAD with stress echocardiography (with cCTA reserved for follow-up of positive stress tests) may be more cost-effective as compared to an initial cCTA for the low risk patient [9].

A minority of cardiac imaging procedures are performed for structural or functional evaluation, unrelated to CAD. For these examinations, echocardiography is a less expensive and less invasive technique compared to cCTA. Transtho- racic echocardiography can be performed portably and does not generally require administration of intravenous contrast material. Transesophageal echocardiography can be performed when there is a need for higher spatial resolution or to visualize parts of the heart-such as the left atrial appendage - that may be difficult to image from the transthoracic approach. Echocardiography is superior to CT when the primary objective of diagnostic imaging is for assessment of ventricular wall motion, valve morphology and function, as well as for quantification of flow velocities and pressure gradients within the heart. Doppler evaluation provides a simple method to grade the severity of valvular stenosis or insufficiency, as well as other functional abnormalities such as tamponade and restrictive physiology. Based upon the superior temporal resolution of echocardiography, echocardiography is often superior to cCTA and MRI for detection of mobile masses and vegetations within the heart.

MRI is limited by spatial resolution for evaluation of coronary artery branches, but is an excellent modality for evaluation of myocardial function, perfusion and viability $[52,53]$. As compared with cCTA, there is no ionizing radiation and no need for a contrast injection to evaluate function with steady-state free-precession sequences. The excellent contrast between the blood pool and the endocardial surface allows clear depiction of cardiac morphology and reproducible measurements of chamber size. When
Fig. 24 Ebstein's anomaly with apical displacement of the septal leaflet of the tricuspid valve (arrowhead), a large, redundant "sail-like" anterior tricuspid leaflet (arrow) and marked enlargement of the right heart. a Four-chamber view. b Short axis view
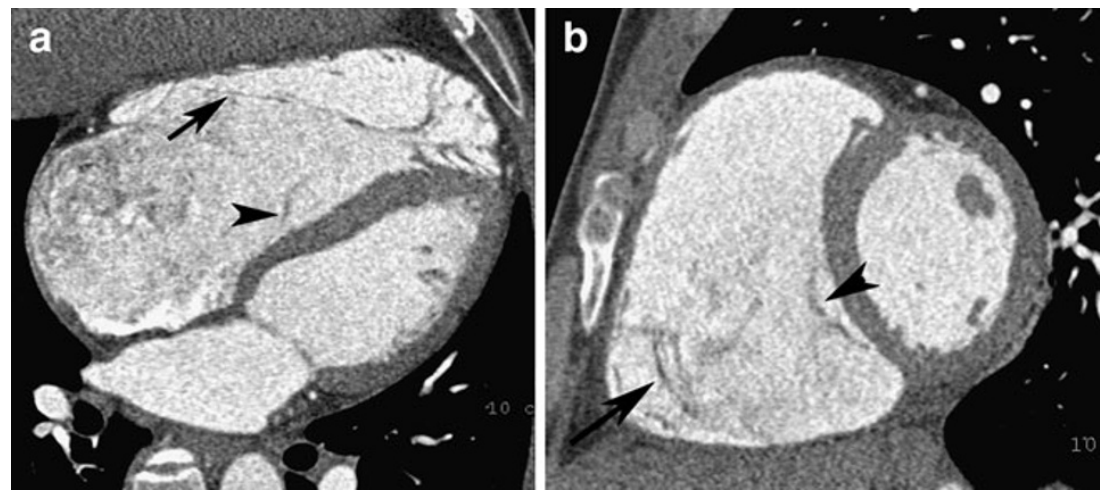


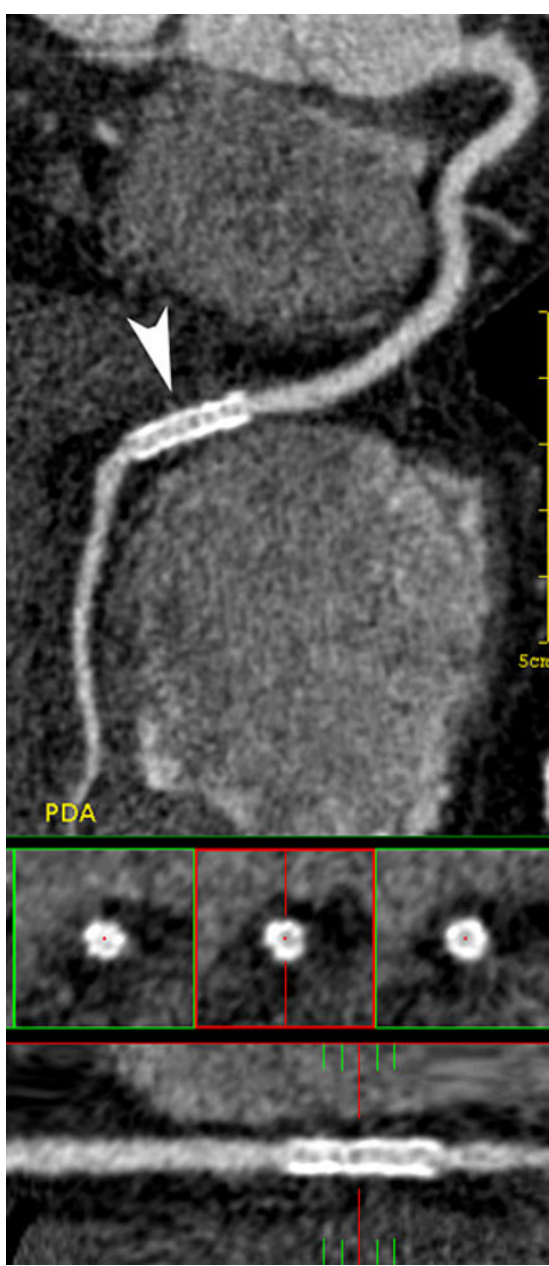

Fig. 25 Curved MIP of the right coronary artery and posterior descending artery. A patent stent is visualized in the distal RCA (arrowhead). There is no significant narrowing of the RCA

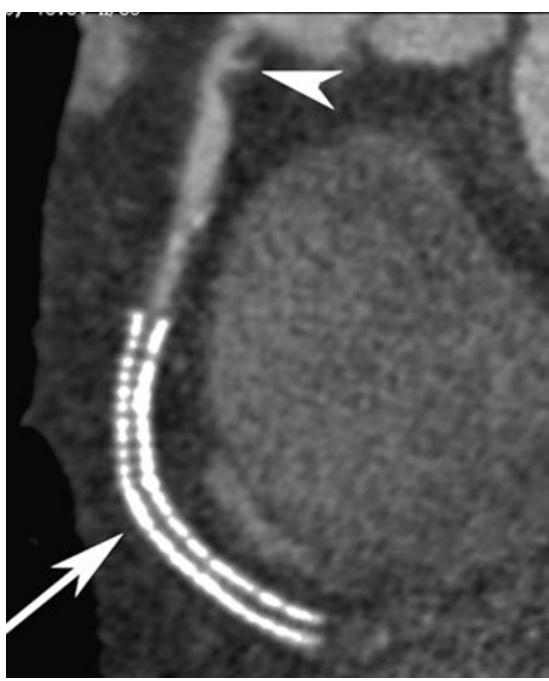

Fig. 26 Slab MIP of the proximal to mid-right coronary artery demonstrates an ulcerated plaque at the origin of the RCA (arrowhead) as well as an occluded stent in the mid-portion of the RCA (arrow) gadolinium is administered, different patterns of myocardial perfusion and delayed enhancement may be used to characterize various types of cardiomypathy and to document the extent of viable tissue. MRI may be combined with pharmacological agents for stress perfusion imaging. The major clinical applications for cardiac MRI at this time include imaging of structural morphology and myocardial evaluation.

\section{Radiation reduction techniques}

A major limitation of cCTA compared with stress echocardiography and MRI is the exposure to ionizing radiation. Various strategies have been implemented in order to reduce this exposure. At the level of CT hardware, there is a constant effort to produce more efficient detectors. At the level of image acquisition, newer techniques have been developed that limit exposure to smaller portions of the cardiac cycle. Finally, at the level of image reconstruction, newer technology is being developed to improve signal to noise in CT images with fewer photons in the raw data.

Conventional cCTA is performed with an ECG-gated helical image acquisition to reconstruct an image during the quiescent phase of the cardiac cycle, usually in mid-diastole. In order to obtain sufficient data for reconstruction, helical scans are performed with a pitch in the range of 0.2-0.3. This low pitch provides sufficient overlap between successive helical rotations of the detector array such that adequate data are obtained in each portion of the cardiac cycle for image reconstruction. Unfortunately, the low pitch also results in relatively high radiation exposure. The data obtained from the quiescent portion of diastole are used to reconstruct highresolution images of the coronary arteries, while data obtained from the remainder of the cardiac cycle are used to reconstruct lower resolution functional images that may be used to demonstrate valve motion and ventricular contraction. However, much of the data acquired during the nonquiescent portions of the cardiac cycle is not needed. A technique known as tube-current modulation has been developed to adjust the current to the $\mathrm{x}$-ray tube such that the full dose is delivered during the quiescent phase of the cardiac cycle for high-resolution imaging, while a much reduced dose is delivered in other phases of the cycle to allow reconstruction of lower resolution functional images. At a heart rate of 60 beats per minute, tube current modulation may result in a dose reduction of greater than $50 \%$ without loss of image quality [54].

Prospective ECG-gating with an axial "step and shoot" technique has been introduced to further reduce radiation dose. This technique acquires images in axial mode during a single, short (usually mid-diastolic) interval. The entire heart is acquired in one or more axial steps (Fig. 7). The 
prospective gating technique relies on a stable heart rate to predict the time for mid-diastole and to trigger the $\mathrm{x}$-ray tube only during that interval for imaging. Evaluation of cardiac motion is not possible with standard step-and-shoot acquisition since image data are not obtained during the systolic portion of the cardiac cycle. Furthermore, image quality may be adversely impacted if a variable heart rhythm results in image acquisition during a suboptimal phase of the cardiac cycle. Nonetheless, a recent multicenter international study included 685 patients, with 99 patients evaluated using prospective ECG-gating and 586 patients evaluated with a standard helical approach. Mean radiation dose was reduced by $68 \%$, from $11.2 \mathrm{mSv}$ for the helical mode to $3.6 \mathrm{mSv}$ for the step-and-shoot technique, without loss of image quality [55].

High pitch spiral acquisition is a new variation on helical scan technology that can be used to acquire cCTA of the entire heart in a single spiral acquisition for patients with a sufficiently low heart rate. Rapid table motion is timed so that the entire heart is imaged during the quiescent phase of a single cardiac cycle. A recent study of 100 patients comparing prospective ECG-gating with step-and-shoot technology to prospective ECG-gating with a high pitch spiral mode (pitch 3.4) demonstrated diagnostic image quality in over $98 \%$ of coronary artery segments with no significant difference in image quality between the two methods [56]. Mean effective radiation dose was $1.4 \pm$ $0.4 \mathrm{mSv}$ for prospective ECG gating with step-and-shoot technology and $0.9 \pm 0.1 \mathrm{mSv}$ for prospective ECG gating with high-pitch spiral mode.

Most contemporary CT scanners utilize a mathematical technique called filtered back projection to reconstruct $\mathrm{CT}$ images. Iterative reconstruction is another category of techniques that has recently gained popularity for reconstruction of CT images with decreased image noise. Iterative reconstruction techniques employ an initial estimate of voxel attenuation to predict projection data; estimates of voxel attenuation are iteratively adjusted to minimize the difference between the predicted projection data and the measured projection data. These techniques are more computationally intensive as compared to filtered back projection, but can result in reduced image noise; alternatively, iterative techniques may be used to maintain an acceptable signal-to-noise ratio and image quality with reduced radiation (Fig. 27). A recent study suggests that iterative techniques may allow a reduction in effective radiation exposure by approximately $50 \%$ [57]. Based upon the doses recorded from studies of prospective ECG-gating and high pitch helical technique, new acquisition techniques combined with iterative reconstruction should routinely provide cCTA imaging with effective radiation exposures of $1 \mathrm{mSv}$ or less.

\section{Conclusion}

Advances in CT technology over the past decade have resulted in marked improvements in cCTA image quality along with reduction in effective radiation dose. We have reviewed the application of ECG-gated CTA of the heart to various clinical applications, including evaluation of the thoracic aorta, aortic valve, bypass grafts, left atrium and various structural cardiac abnormalities. However, the major indication for cardiac CT is cCTA for evaluation of CAD in symptomatic patients with low to intermediate risk of CAD. High-risk symptomatic patients should be triaged directly to cardiac catheterization. In patients with a risk of CAD below $20 \%$, stress echocardiography may present the most costeffective study for initial evaluation. cCTA is useful in these patients to follow-up on positive stress tests in order to avoid catheterization of patients with false-positive stress test results. As the pre-test probability of CAD in a symptomatic patient rises above $20 \%$, cCTA provides a cost-effective initial evaluation. Stress perfusion imaging may be useful as a secondary study in these patients when moderate stenosis
Fig. 27 Iterative reconstruction illustrated for a curved MIP of a normal RCA scanned with very low dose technique. a Standard reconstruction with filtered back projection demonstrates a noisey image related to inadequate signal-to-noise characteristics. b Iterative reconstruction using iDose (Philips Medical Systems) with the same raw data demonstrates improved signal-to-noise characteristics with improved definition of the smaller distal RCA branches
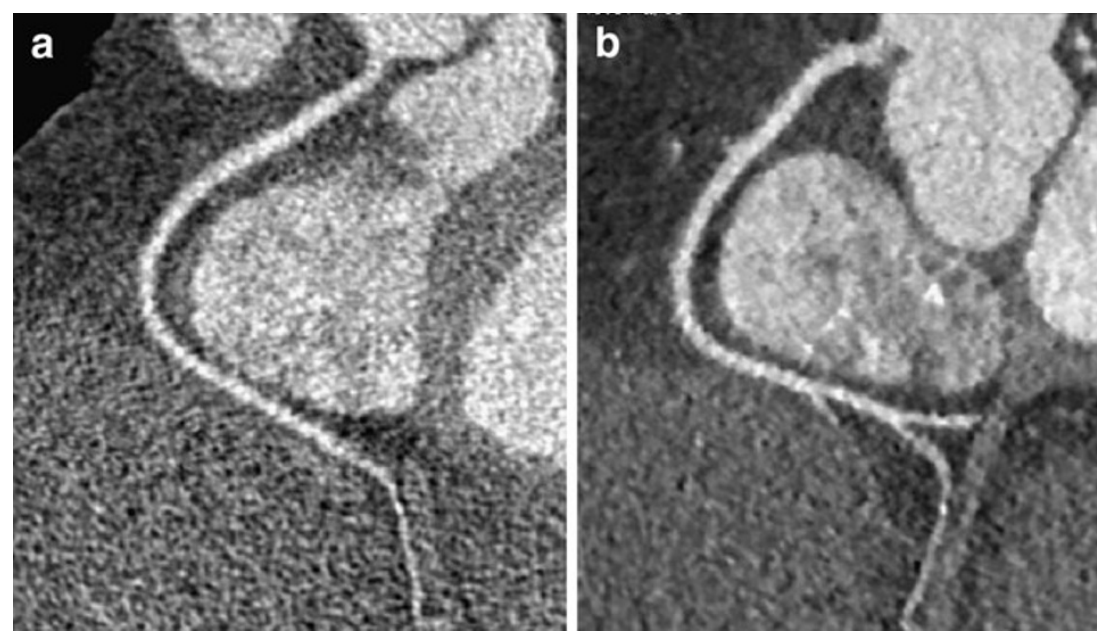
(50-75\% diameter reduction) is identified on cCTA. New developments that allow myocardial perfusion imaging with cCTA are likely to result in a single comprehensive CT examination for CAD in the future.

\section{References}

1. Heron M, Hoyert DL, Murphy SL, Xu J, Kochanek KD, TejadaVera B (2009) National Vital Statistics Reports-Deaths: final data for 2006. Vol 57, No. 14. April 2009. Centers for Disease Control and Prevention. http://www.cdc.gov/nchs/data/nvsr/nvsr57/ nvsr57_14.pdf

2. Stein PD, Yaekoub AY, Matta F et al (2008) Sixty-four-slice CT for diagnosis of coronary artery disease: a systematic review. Am J Med 121(8):715-725

3. Mowatt G, Cook JA, Hillis GS et al (2008) Sixty-four-slice computed tomography angiography in the diagnosis and assessment of coronary artery disease: systematic review and metaanalysis. Heart 94(11):1386-1393

4. Abdulla J, Abildstrom SZ, Gotzsche O et al (2007) Sixty-fourmultislice detector computed tomography coronary angiography as potential alternative to conventional coronary angiography: a systematic review and meta-analysis. Eur Heart J 28(24):30423050

5. Gopalakrishnan P, Wilson GT, Tak T (2008) Accuracy of multislice computed tomography coronary angiography: a pooled estimate. Cardiol Rev 16(4):189-196

6. Hacker M, Jakobs T, Hack N, Nikolaou K, Becker C, von Ziegler F, Knez A, König A, Klauss V, Reiser M, Hahn K, Tiling R (2007) Sixty-four-slice spiral CT angiography does not predict the functional relevance of coronary artery stenoses in patients with stable angina. Eur J Nucl Med Mol Imaging 34(1):4-10

7. Gaemperli O, Schepis T, Valenta I, Koepfli P, Husmann L, Scheffel H, Leschka S, Eberli FR, Luscher TF, Alkadhi H, Kaufmann PA (2008) Functionally relevant coronary artery disease: comparison of 64-section CT angiography with myocardial perfusion SPECT. Radiology 248(2):414-423

8. Schuijf JD, Bax JJ (2008) CT angiography: an alternative to nuclear perfusion imaging? Heart 94(3):255-257

9. Halpern EJ, Fischman D, Savage M, Koka AR, DeCaro M, Levin DC (2010) Decision analytic model for evaluation of suspected coronary disease with stress testing and coronary CT angiography. Acad Radiol 17(5):577-586

10. Diamond GA, Forrester JS (1979) Analysis of probability as an aid in the clinical diagnosis of coronary-artery disease. N Engl J Med 300 (24): $1350-1358$

11. Goldstein JA, Gallagher MJ, O'Neill WW, Ross MA, O'Neil BJ, Raff GL (2007) A randomized controlled trial of multi-slice coronary computed tomography for evaluation of acute chest pain. J Am Coll Cardiol 49(8):863-871

12. Ladapo JA, Hoffmann U, Bamberg F, Nagurney JT, Cutler DM, Weinstein MC, Gazelle GS (2008) Cost-effectiveness of coronary MDCT in the triage of patients with acute chest pain. AJR Am J Roentgenol 191(2):455-463

13. Halpern EJ (2009) Triple-rule-out CT angiography for evaluation of acute chest pain and possible acute coronary syndrome. Radiology 252(2):332-345

14. Halpern EJ, Levin DC, Zhang S, Takakuwa KM (2009) Comparison of image quality and arterial enhancement with a dedicated coronary CTA protocol versus a triple rule-out coronary CTA protocol. Acad Radiol 16(9):1039-1048

15. Hendel RC, Patel MR, Kramer CM, Poon M, Hendel RC, Carr JC, Gerstad NA, Gillam LD, Hodgson JM, Kim RJ, Kramer CM,
Lesser JR, Martin ET, Messer JV, Redberg RF, Rubin GD, Rumsfeld JS, Taylor AJ, Weigold WG, Woodard PK, Brindis RG, Hendel RC, Douglas PS, Peterson ED, Wolk MJ, Allen JM, American College of Cardiology Foundation Quality Strategic Directions Committee Appropriateness Criteria Working Group, American College of Radiology, Society of Cardiovascular Computed Tomography, Society for Cardiovascular Magnetic Resonance, American Society of Nuclear Cardiology, North American Society for Cardiac Imaging, Society for Cardiovascular Angiography and Interventions, Society of Interventional Radiology (2006) ACCF/ACR/SCCT/SCMR/ASNC/NASCI/ SCAI/SIR 2006 appropriateness criteria for cardiac computed tomography and cardiac magnetic resonance imaging: a report of the American College of Cardiology Foundation Quality Strategic Directions Committee Appropriateness Criteria Working Group, American College of Radiology, Society of Cardiovascular Computed Tomography, Society for Cardiovascular Magnetic Resonance, American Society of Nuclear Cardiology, North American Society for Cardiac Imaging, Society for Cardiovascular Angiography and Interventions, and Society of Interventional Radiology. J Am Coll Cardiol 48(7):1475-1497

16. Halpern EJ, Savage MP, Fischman DL, Levin DC (2010) Costeffectiveness of coronary $\mathrm{CT}$ angiography in evaluation of patients without symptoms who have positive stress test results. AJR Am J Roentgenol 194(5):1257-1262

17. Kondos GT, Hoff JA, Sevrukov A et al (2003) Electron-beam tomography coronary artery calcium and cardiac events: a 37-month follow-up of 5635 initially asymptomatic low- to intermediate-risk adults. Circulation 107:2571-2576

18. Shaw LJ, Raggi P, Schisterman E, Berman DS, Callister TQ (2003) Prognostic value of cardiac risk factors and coronary artery calcium screening for all-cause mortality. Radiology 228:826-833

19. Budoff MJ, Shaw LJ, Liu ST et al (2007) Long-term prognosis associated with coronary calcification: observations from a registry of 25, 253 patients. J Am Coll Cardiol 49:1860-1870

20. Greenland P, LaBree L, Azen SP, Doherty TM, Detrano RC (2004) Coronary artery calcium score combined with Framingham score for risk prediction in asymptomatic individuals. JAMA 291:210-215

21. Arad Y, Goodman KJ, Roth M, Newstein D, Guerci AD (2005) Coronary calcification, coronary disease risk factors, C-reactive protein, and atherosclerotic cardiovascular disease events: the St. Francis Heart Study. J Am Coll Cardiol 46:158-165

22. Marwan M, Ropers D, Pflederer T, Daniel WG, Achenbach S (2009) Clinical characteristics of patients with obstructive coronary lesions in the absence of coronary calcification: an evaluation by coronary CT angiography. Heart 95:1056-1960

23. Gottlieb I, Miller JM, Arbab-Zadeh A, Dewey M, Clouse ME, Sara L, Niinuma H, Bush DE, Paul N, Vavere AL, Texter J, Brinker J, Lima JA, Rochitte CE (2010) The absence of coronary calcification does not exclude obstructive coronary artery disease or the need for revascularization in patients referred for conventional coronary angiography. J Am Coll Cardiol 55:627-634

24. Abdulla J, Asferg C, Kofoed KF (2010) Prognostic value of absence or presence of coronary artery disease determined by 64 slice computed tomography coronary angiography A systematic review and meta-analysis. Int J Cardiovasc Imaging Jun 12. [Epub ahead of print]

25. Pundziute G, Schuijf JD, Jukema JW, Boersma E, de Roos A, van der Wall EE, Bax JJ (2007) Prognostic value of multislice computed tomography coronary angiography in patients with known or suspected coronary artery disease. J Am Coll Cardiol 49(1):62-70

26. Achenbach S, Raggi $P$ (2010) Imaging of coronary atherosclerosis by computed tomography. Eur Heart J 31(12):1442-1448

27. Hachamovitch R, Hayes SW, Friedman JD, Cohen I, Berman DS (2003) Comparison of the short-term survival benefit associated 
with revascularization compared with medical therapy in patients with no prior coronary artery disease undergoing stress myocardial perfusion single photon emission computed tomography. Circulation 107(23):2900-2907

28. Hachamovitch R, Kang X, Amanullah AM, Abidov A, Hayes SW, Friedman JD, Cohen I, Thomson LE, Germano G, Berman DS (2009) Prognostic implications of myocardial perfusion singlephoton emission computed tomography in the elderly. Circulation 120(22):2197-2206

29. Ko SM, Seo JB, Hong MK, Do KH, Lee SH, Lee JS, Song JW, Park SJ, Park SW, Lim TH (2006) Myocardial enhancement pattern in patients with acute myocardial infarction on two-phase contrast-enhanced ECG-gated multidetector-row computed tomography. Clin Radiol 61(5):417-422

30. Kopp AF, Heuschmid M, Reimann A, Kuettner A, Beck T, Ohmer M, Burgstahler C, Brodoefel H, Claussen CD, Schroeder S (2005) Evaluation of cardiac function and myocardial viability with 16and 64-slice multidetector computed tomography. Eur Radiol 4: D15-D20

31. Nagao M, Matsuoka H, Kawakami H, Higashino H, Mochizuki T, Murase K, Uemura M (2008) Quantification of myocardial perfusion by contrast-enhanced 64-MDCT: characterization of ischemic myocardium. AJR Am J Roentgenol 191(1):19-25

32. Nagao M, Matsuoka H, Kawakami H, Higashino H, Mochizuki T, Ohshita A, Kohno T, Shigemi S (2009) Detection of myocardial ischemia using 64-slice MDCT. Circ J 73(5):905-911

33. George RT, Arbab-Zadeh A, Miller JM, Kitagawa K, Chang HJ, Bluemke DA, Becker L, Yousuf O, Texter J, Lardo AC, Lima JA (2009) Adenosine stress 64- and 256-row detector computed tomography angiography and perfusion imaging: a pilot study evaluating the transmural extent of perfusion abnormalities to predict atherosclerosis causing myocardial ischemia. Circ Cardiovasc Imaging 2(3): 174-182

34. Okada DR, Ghoshhajra BB, Blankstein R, Rocha-Filho JA, Shturman LD, Rogers IS, Bezerra HG, Sarwar A, Gewirtz H, Hoffmann U, Mamuya WS, Brady TJ, Cury RC (2010) Direct comparison of rest and adenosine stress myocardial perfusion CT with rest and stress SPECT. J Nucl Cardiol 17(1):27-37

35. Blankstein R, Shturman LD, Rogers IS, Rocha-Filho JA, Okada DR, Sarwar A, Soni AV, Bezerra H, Ghoshhajra BB, Petranovic M, Loureiro R, Feuchtner G, Gewirtz H, Hoffmann U, Mamuya WS, Brady TJ, Cury RC (2009) Adenosine-induced stress myocardial perfusion imaging using dual-source cardiac computed tomography. J Am Coll Cardiol 54(12):1072-1084

36. Ruzsics B, Lee H, Zwerner PL, Gebregziabher M, Costello P, Schoepf UJ (2008) Dual-energy CT of the heart for diagnosing coronary artery stenosis and myocardial ischemia-initial experience. Eur Radiol 18(11):2414-2424

37. Ruzsics B, Schwarz F, Schoepf UJ, Lee YS, Bastarrika G, Chiaramida SA, Costello P, Zwerner PL (2009) Comparison of dual-energy computed tomography of the heart with single photon emission computed tomography for assessment of coronary artery stenosis and of the myocardial blood supply. Am J Cardiol 104 (3):318-326

38. Baile EM, Paré PD, D'yachkova Y, Carere RG (1999) Effect of contrast media on coronary vascular resistance: contrast-induced coronary vasodilation. Chest 116(4):1039-1045

39. Kim SY, Seo JB, Do KH, Heo JN, Lee JS, Song JW, Choe YH, Kim TH, Yong HS, Choi SI, Song KS, Lim TH (2006) Coronary artery anomalies: classification and ECG-gated multi-detector row CT findings with angiographic correlation. Radiographics 26:317-333

40. Herzog C, Wimmer-Greinecker G, Schwarz W, Dogan S, Moritz A, Fichtlscherer S, Vogl TJ (2004) Progress in CT imaging for the cardiac surgeon. Semin Thorac Cardiovasc Surg 16:242-248

41. Gasparovic H, Rybicki FJ, Millstine J, Unic D, Byrne JG, Yucel K, Mihaljevic T (2005) Three dimensional computed tomographic imaging in planning the surgical approach for redo cardiac surgery after coronary revascularization. Eur J Cardiothorac Surg 28:244-249

42. Halpern EJ, Mallya R, Sewell M, Shulman M, Zwas DR (2009) Differences in aortic valve area measured with CT planimetry and echocardiography (continuity equation) are related to divergent estimates of left ventricular outflow tract area. AJR Am J Roentgenol 192(6):1668-1673

43. Bollati M, Tizzani E, Moretti C, Sciuto F, Omede P, Zoccai GB, Trevi GP, Abbate A, Sheiban I (2010) The future of new aortic valve replacement approaches. Future Cardiol 6(3):351-360

44. Rivard AL, Bartel T, Bianco RW, O'Donnell KS, Bonatti J, Dichtl W, Cury RC, Feuchtner GM (2009) Evaluation of aortic root and valve calcifications by multi-detector computed tomography. J Heart Valve Dis 18(6):662-670

45. Lentini S, Monaco F, Tancredi F, Savasta M, Gaeta R (2009) Aortic valve infective endocarditis: could multi-detector CT scan be proposed for routine screening of concomitant coronary artery disease before surgery? Ann Thorac Surg 87(5):1585-1587

46. Tops LF, Schalij MJ (2008) Multislice CT: is it essential before atrial fibrillation ablation? Heart 94(8):973-975

47. Halpern EJ. Clinical cardiac CT: anatomy and function. 2nd ed. New York: Thieme, 2011 (in press)

48. Gianrossi R, Detrano R, Mulvihill D, Lehmann K, Dubach P, Colombo A, McArthur D, Froelicher V (1989) Exercise-induced ST depression in the diagnosis of coronary artery disease. A metaanalysis. Circulation 80(1):87-98

49. Schinkel AF, Bax JJ, Geleijnse ML et al (2003) Noninvasive evaluation of ischaemic heart disease: myocardial perfusion imaging or stress echocardiography? Eur Heart J 24:789e800

50. Mahajan N, Polavaram L, Vankayala H, Ference B, Wang Y, Ager J, Kovach J, Afonso L (2010) Diagnostic accuracy of myocardial perfusion imaging and stress echocardiography for the diagnosis of left main and triple vessel coronary artery disease: a comparative meta-analysis. Heart 96(12):956-966

51. Yao SS, Bangalore S, Chaudhry FA (2010) Prognostic implications of stress echocardiography and impact on patient outcomes: an effective gatekeeper for coronary angiography and revascularization. J Am Soc Echocardiogr May 27. [Epub ahead of print]

52. Dembo LG, Shifrin RY, Wolff SD (2004) MR imaging in ischemic heart disease. Radiol Clin North Am 42(3):651-673, vii

53. Reeder SB, Du YP, Lima JA, Bluemke DA (2001) Advanced cardiac MR imaging of ischemic heart disease. Radiographics 21 (4):1047-1074

54. Takakuwa KM, Halpern EJ, Gingold EL, Levin DC, Shofer FS (2009) Radiation dose in a "triple rule-out" coronary CT angiography protocol of emergency department patients using 64-MDCT: the impact of ECG-based tube current modulation on age, sex, and body mass index. AJR Am J Roentgenol 192 (4): $866-872$

55. Bischoff B, Hein F, Meyer T, Krebs M, Hadamitzky M, Martinoff S, Schömig A, Hausleiter J (2010) Comparison of sequential and helical scanning for radiation dose and image quality: results of the Prospective Multicenter Study on Radiation Dose Estimates of Cardiac CT Angiography (PROTECTION) I Study. AJR Am J Roentgenol 194(6):1495-1499

56. Alkadhi H, Stolzmann P, Desbiolles L, Baumueller S, Goetti R, Plass A, Scheffel H, Feuchtner G, Falk V, Marincek B, Leschka S (2010) Low-dose, 128-slice, dual-source CT coronary angiography: accuracy and radiation dose of the high-pitch and the stepand-shoot mode. Heart 96(12):933-938

57. Gosling O, Loader R, Venables P, Roobottom C, Rowles N, Bellenger N, Morgan-Hughes G (2010) A comparison of radiation doses between state-of-the-art multislice CT coronary angiography with iterative reconstruction, multislice CT coronary angiography with standard filtered back-projection and invasive diagnostic coronary angiography. Heart 96(12):922-926 